Wilfrid Laurier University

Scholars Commons @ Laurier

2004

\title{
Weight modules over generalized Witt algebras with 1-dimensional weight spaces
}

Kaiming Zhao

Wilfrid Laurier University, kzhao@wlu.ca

Follow this and additional works at: https://scholars.wlu.ca/math_faculty

\section{Recommended Citation}

Zhao, Kaiming, "Weight modules over generalized Witt algebras with 1-dimensional weight spaces" (2004). Mathematics Faculty Publications. 49.

https://scholars.wlu.ca/math_faculty/49

This Article is brought to you for free and open access by the Mathematics at Scholars Commons @ Laurier. It has been accepted for inclusion in Mathematics Faculty Publications by an authorized administrator of Scholars Commons@Laurier. For more information, please contact scholarscommons@wlu.ca. 


\title{
Weight modules over generalized Witt algebras with 1-dimensional weight spaces ${ }^{1}$
}

Kaiming Zhao

(Communicated by Karl Hermann Neeb)

\begin{abstract}
In this paper, indecomposable and irreducible weight representations with 1 dimensional weight spaces for simple generalized Witt algebras over any field of characteristic 0 are classified. There are five classes of such nontrivial indecomposable modules.
\end{abstract}

2000 Mathematics Subject Classification: 17B10, 17B20, 17B65, 17B66, $17 \mathrm{~B} 68$.

\section{§1 Introduction}

Throughout this paper we use the definitions and notations in [DZ1], and assume that $F$ is an arbitrary field of characteristic 0 . Let me start with the definition of generalized Witt algebras.

Let $A$ be an abelian group, $T$ a vector space over $F$. We denote by $F[A]$ the group algebra of $A$ over $F$. The elements $t^{x}, x \in A$, form a basis of $F[A]$ with the multiplication $t^{x} t^{y}=t^{x+y}$. We shall write 1 instead of $t^{0}$. The tensor product $W=F[A] \otimes_{F} T$ is a free left $F[A]$-module. We shall usually denote an arbitrary element of $T$ by $\partial$, and for simplicity we write $t^{x} \partial$ instead of $t^{x} \otimes \partial$. We now fix a pairing $\varphi: T \times A \rightarrow$ $F$, which is $F$-linear in the first variable and additive in the second one. For convenience we shall also use the following notations:

$$
\varphi(\partial, x)=\partial(x)=x(\partial) \text { for any } \partial \in T \text { and } x \in A \text {. }
$$

Under the bracket

$$
\left[t^{x} \partial_{1}, t^{y} \partial_{2}\right]:=t^{x+y}\left(\partial_{1}(y) \partial_{2}-\partial_{2}(x) \partial_{1}\right), \quad \text { for any } \partial_{1}, \partial_{2} \in T \text { and } x, y \in A,
$$

$W$ becomes a Lie algebra. We shall refer to $W=W(A, T, \varphi)$ as a generalized Witt algebra. Generalized Witt algebras were thoroughly studied in [DZ1]. For further

${ }^{1}$ This work is supported by Hundred Talents Program of Chinese Academy of Sciences and by NSF of China. 
generalizations of this class of Lie algebras, please refer to [DZ2], [Jo], [P], and $[\mathrm{X}]$, where Lie algebras not belonging to $W(A, T, \varphi)$ generally do not have a torus. The structure theory of generalized Witt algebras is quite complete.

From [DZ1] we know that $W$ is a simple Lie algebra if and only if $A$ is nonzero and $\varphi$ is nondegenerate in the sense that $\varphi(x, A)=0$ and $\varphi(A, y)=0$ implies $x=0$ and $y=0$ respectively. In this case $A$ must be torsion-free.

When $A \simeq \mathbb{Z}^{n}$ and $\operatorname{dim} T=n$, the simple Lie algebra $W(A, T, \varphi)$ is isomorphic to the classical Witt algebra $W_{n}=\operatorname{Der}\left(F\left[t_{1}^{ \pm 1}, \ldots t_{n}^{ \pm 1}\right]\right)$, where $F\left[t_{1}^{ \pm 1}, \ldots t_{n}^{ \pm 1}\right]$ is the Laurent polynomial algebra. When $\operatorname{dim} T=1$ ( $A$ arbitrary), the simple Lie algebra $W(A, T, \varphi)$ is isomorphic to a centerless generalized Virasoro algebra introduced in [PZ] (a generalization of the centerless Virasoro algebra).

For representation theory, we have the complete classification for irreducible weight modules (with finite dimensional weight spaces) over the Virasoro algebra, see [M]. All weight modules with 1-dimensional weight spaces over generalized Virasoro algebras were obtained in [SZ], while the corresponding classification for the classical Virasoro algebra was given in $[\mathrm{Kp}]$. Eswara Rao [E1, E2] and Jia [J] constructed some weight modules over $W_{n}$. In the study of toroidal Lie algebras, a particular case of current algebras, representations of generalized Witt algebras play an important role, see $[\mathrm{BB}]$.

We shall now describe the contents of this paper. We assume that $W=W(A, T, \varphi)$ is always simple.

In Section 2, for convenience of the reader, we collect some known results for later use. In Section 3, we construct five classes of indecomposable weight modules over $W$ with 1-dimensional weight spaces, give the necessary and sufficient conditions for two such modules to be isomorphic, and prove an important result on the module structure. This is different from that for generalized Virasoro algebras where we have only three classes. Also conditions for two such modules for $\operatorname{dim} T>2$ to be isomorphic are different from that for $\operatorname{dim} T=1$ (the case of generalized Virasoro algebras). See Theorem 3.6. When $W \simeq W_{n}$, the first class of these modules are essentially the ones constructed in [E1, E2] and [J].

In Section 4, we classify indecomposable, and irreducible $W$-modules with 1dimensional weight spaces for algebraically closed field $F$ of characteristic 0 (Theorem 4.1). We first used the classification of weight modules with 1-dimensional weight spaces over generalized Virasoro algebras obtained in $[\mathrm{SZ}]$ to prove this result for the critical case of $W_{2}$ (Claim 2 in the proof) and then use the sewing technique (stitch small pieces together) used in [SZ] to obtain the complete classification. The reason we confine our argument on an algebraically closed field is that the proof of Claim 2 (and some other places) requires the field big enough to get a centerless generalized Virasoro algebra.

Then in Section 5 we easily give the classification of indecomposable and irreducible $W$-modules with 1-dimensional weight spaces for arbitrary field $F$ of characteristic 0 (Theorems 5.2 and 5.3).

Here I would like to thank the referee for some suggestions to smooth this paper. 


\section{\$2 Relevant known results}

The Lie algebra $W=W(A, T, \varphi)$ defined in Section 1 has a natural $A$-gradation $W=\bigoplus_{x \in A} W_{x}$ where $W_{x}=t^{x} T$ for $x \in A$. In particular we have $W_{0}=T$. It follows that

$$
\left[\partial, t^{x} \partial_{1}\right]=\partial(x) t^{x} \partial_{1}, \quad \forall \partial, \partial_{1} \in T, x \in A,
$$

i.e., $\operatorname{ad}(\partial)$ acts on $W_{x}$ as the scalar $\partial(x)$. Hence, $\partial$ is semisimple in the sense that $\operatorname{ad}(\partial)$ is a semisimple operator on $W$. Consequently, $T$ is a (actually the unique) torus, i.e., the maximal abelian subalgebra of $W$ consisting of semisimple elements.

In this paper we are only interested in simple algebras $W(A, T, \varphi)$, that is, we assume that $A$ is nonzero (torsion-free) and $\varphi$ is nondegenerate. If $\operatorname{dim} T=1$, the simple algebra $W(A, T, \varphi)$ is essentially the centerless generalized Virasoro algebra. Now I am going to present this algebra in another notation used in [SZ].

Let $M$ be an abelian additive sub-group of $F$. The centerless generalized Virasoro algebra $\operatorname{Vir}[M]$ is defined to be the Lie algebra with $F$-basis $\left\{L_{\mu}, c \mid \mu \in M\right\}$, subject to the following commutator relations:

$$
\left[L_{\mu}, L_{v}\right]=(v-\mu) L_{\mu+v}, \quad \text { for any } \mu, v \in M .
$$

For any $a, b \in F$, in [SZ] the following three series of $\operatorname{Vir}[M]$-modules $\mathscr{A}_{a, b}(M)$, $\mathscr{A}_{a}(M), \mathscr{B}_{a}(M)$ were defined: they all have basis $\left\{v_{\mu} \mid \mu \in M\right\}$ with actions given by the following formulas

$$
\begin{aligned}
& \mathscr{A}_{a, b}(M): L_{\mu} v_{v}=(a+v+\mu b) v_{\mu+v}, \\
& \mathscr{A}_{a}(M): L_{\mu} v_{v}=v v_{\mu+v}, \text { if } v(\mu+v) \neq 0 ; \quad L_{\mu} v_{\mu}=0, \quad L_{\mu} v_{0}=(a \mu+1) v_{\mu}, \\
& \mathscr{B}_{a}(M): L_{\mu} v_{v}=(\mu+v) v_{\mu+v}, \text { if } v(\mu+v) \neq 0 ; \quad L_{\mu} v_{0}=0, \quad L_{\mu} v_{\mu}=-(a \mu+1) v_{0},
\end{aligned}
$$

for any $\mu, v \in M$. We use $\mathscr{A}_{a, b}^{\prime}(M), \mathscr{A}_{a}^{\prime}(M), \mathscr{B}_{a}^{\prime}(M)$ to denote the nontrivial irreducible sub-quotient of $\mathscr{A}_{a, b}(M), \mathscr{A}_{a}(M), \mathscr{B}_{a}(M)$, respectively. We know that $\mathscr{A}_{a, b}^{\prime}(M) \neq \mathscr{A}_{a, b}(M)$ if and only if $a \in M$ and $b \in\{0,1\}$.

Theorem 2.1 ([SZ, Theorem 4.1]). Among the $\operatorname{Vir}[M]$-modules $\mathscr{A}_{a, b}(M), \mathscr{A}_{a}(M)$, $\mathscr{B}_{a}(M)$ for $a, b \in F$, and their nontrivial sub-quotients, we have only the following module isomorphisms:

(i) $\mathscr{A}_{a, b}(M) \simeq \mathscr{A}_{a^{\prime}, b}(M)$ if $a-a^{\prime} \in M$,

(ii) $\mathscr{A}_{a, 0}(M) \simeq \mathscr{A}_{a^{\prime}, 1}(M)$ for $a \notin M$ with $a-a^{\prime} \in M$,

(iii) $\mathscr{A}_{a, b}^{\prime}(M) \simeq \mathscr{A}_{a^{\prime}, b}^{\prime}(M)$ if $a-a^{\prime} \in M$,

(iv) $\mathscr{A}_{a, 0}^{\prime}(M) \simeq \mathscr{A}_{a^{\prime}, 1}^{\prime}(M)$ if $a-a^{\prime} \in M$,

(v) $\quad \mathscr{A}_{a}^{\prime}(M) \simeq \mathscr{B}_{b}^{\prime}(M) \simeq \mathscr{A}_{0,0}^{\prime}(M)$. 
Theorem 2.2. Let $V=\bigoplus_{j \in M} V_{a+j}$ be a $\operatorname{Vir}[M]$-module with $\operatorname{dim} V_{a+j} \leq 1$ for all $j \in M$, where $a \in F$ and $V_{\alpha}=\left\{v \in V \mid L_{0} \cdot v=\alpha v\right\}$. Then $V$ is isomorphic to one of the following for appropriate $a, \beta \in F$ :

(i) $\mathscr{A}_{a, \beta}^{\prime}(M)$,

(ii) $\mathscr{A}_{0,0}^{\prime}(M) \oplus F v_{0}$ as direct sum of $\operatorname{Vir}(M)$-modules,

(iii) $\mathscr{A}_{\beta}(M)$,

(iv) $\mathscr{B}_{\beta}(M)$,

(v) $\mathscr{A}_{0,0}(M), \mathscr{A}_{0,1}(M)$,

(vi) $F v_{0}$.

The proof here is the same as that of Theorem 4.5 of [SZ] just replacing [2, Theorem 2.1] there by [SZ, Theorem 4.6] in that proof. We omit the details.

\section{\$3 Weight modules with weight multiplicity 1}

In this section we shall define five classes of indecomposable $W$-modules: $\mathscr{A}_{\alpha, b}(W)$, $\mathscr{A}_{\alpha}(W), \tilde{\mathscr{A}}_{\alpha}(W), \mathscr{B}_{\alpha}(W), \tilde{\mathscr{B}}_{\alpha}(W)$ for $\alpha \in T^{*}, b \in F$.

Recall that we have assumed that $W$ is simple. Since $\varphi$ is nondegenerate we can regard $A$ as a sub-group of the dual space $T^{*}=\operatorname{Hom}(T, F)$ via $\varphi$, i.e., $x(\partial)=\varphi(\partial, x)$, $\forall x \in A, \partial \in T$. A weight module over $W(A, T, \varphi)$ is a module $V=\bigoplus_{\alpha \in T^{*}} V_{\alpha}$, where $V_{\alpha}=\{v \in V \mid \partial \cdot v=\alpha(\partial) v \forall \partial \in T\}$ which is called the weight space with weight $\alpha \in T^{*}$. The integer $\operatorname{dim} V_{\alpha}$ is called the multiplicity of $\alpha$.

For any $\alpha \in T^{*}$ and $b \in F$, we define the $A$-graded $W$-module $\mathscr{A}_{\alpha, b}(W)=\bigoplus_{x \in A} F v_{x}$ with the action

$$
\left(t^{x} \partial\right) v_{y}=\partial(y+\alpha+b x) v_{x+y}, \quad \text { for all } x, y \in A, \partial \in T \text {. }
$$

Remark. Clearly $\mathscr{A}_{\alpha, b}(W) \simeq \mathscr{A}_{\alpha+x, b}(W)$ for any $x \in A$. In the case of $A=\mathbb{Z}^{n}$ and $\operatorname{dim} T=n$, the modules $\mathscr{A}_{\alpha, b}(W)$ were constructed in [E1, E2] and [J].

Similar to [E1, Proposition 4.1] or [J, Theorem 3.1] we have the following statement:

Lemma 3.1. $\mathscr{A}_{\alpha, b}(W)$ is reducible if and only if $\alpha \in A$ and $b \in\{0,1\}$.

If $\alpha \in A$ and $b=0$, then $F v{ }_{\alpha}$ is the only nontrivial sub-module of $\mathscr{A}_{\alpha, b}$. If $\alpha \in A$ and $b=1$, then $\bigoplus_{x \in A \backslash\left\{{ }_{\alpha}\right\}} F v_{x}$ is the only nontrivial irreducible sub-module of $\mathscr{A}_{\alpha, b}$.

We use $\mathscr{A}_{\alpha b}^{\prime}(W)$ to denote the nontrivial irreducible sub-quotient of $\mathscr{A}_{\alpha, b}(W)$, i.e., $\quad \mathscr{A}_{\alpha, b}^{\prime}(W)=\mathscr{A}_{\alpha, b}(W)$ if $(\alpha, b) \notin(A,\{0,1\}), \quad \mathscr{A}_{0,0}^{\prime}(W)=\frac{\mathscr{A}_{0,0}(W)}{F v_{0}}, \quad \mathscr{A}_{0,1}^{\prime}(W)=$ $\bigoplus_{x \in A \backslash\{0\}} F v_{x}$.

Proposition 3.2. Suppose $\operatorname{dim} T \geq 2$. 
(a) $\mathscr{A}_{\alpha, b}(W) \simeq \mathscr{A}_{\alpha^{\prime}, b^{\prime}}(W)$ if and only if $\alpha-\alpha^{\prime} \in A$ and $b=b^{\prime}$.

(b) $\mathscr{A}_{\alpha, b}^{\prime}(W) \simeq \mathscr{A}_{\alpha^{\prime}, b^{\prime}}^{\prime}(W)$ if and if $\alpha-\alpha^{\prime} \in A$ and $b=b^{\prime}$.

Proof. (a) " $\Leftarrow$ ". This is clear.

“ $\Rightarrow "$. Suppose $\mathscr{A}_{\alpha, b}(W) \simeq \mathscr{A}_{\alpha^{\prime}, b^{\prime}}(W)$. Denote $\mathscr{A}_{\alpha, b}(W)=\bigoplus_{x \in A} F v_{x}, \mathscr{A}_{\alpha^{\prime}, b^{\prime}}^{\prime}(W)=$ $\bigoplus_{x \in A} F v_{x}^{\prime}$ with actions in (3.1). Then $\alpha-\alpha^{\prime} \in A$. From the above remark we may assume that $\alpha=\alpha^{\prime}$, so we have an isomorphism of the form

$$
\theta: \mathscr{A}_{\alpha, b}(W) \rightarrow \mathscr{A}_{\alpha^{\prime}, b^{\prime}}(W), \quad v_{x} \rightarrow f(x) v_{x}^{\prime}
$$

where $f(x) \in F^{*}$ with $f(0)=1$. From $t^{y} \partial \theta\left(v_{x}\right)=\theta\left(\left(t^{y} \partial\right) v_{x}\right)$, it follows that

$$
f(x) \partial\left(x+\alpha+b^{\prime} y\right) v_{x+y}^{\prime}=\partial(x+\alpha+b y) f(x+y) v_{x+y}^{\prime},
$$

i.e.,

$$
\partial(x+\alpha+b y) f(x+y)=f(x)\left(\partial\left(x+\alpha+b^{\prime} y\right)\right), \quad \text { for all } x, y \in A, \partial \in T .
$$

At $x=0,(3.2)$ gives

$$
\partial(\alpha+b y) f(y)=\partial\left(\alpha+b^{\prime} y\right) \text { for all } y \in A, \partial \in T .
$$

If $\alpha=0$, (3.3) yields $b \partial(y) f(y)=b^{\prime} \partial(y)$, for all $\partial \in T, y \in A$. Then $b=0$ if and only if $b^{\prime}=0$. Suppose $b b^{\prime} \neq 0$, then we have $b f(y)=b^{\prime}$ if $y \in A \backslash \operatorname{ker}(\partial)$ for some $\partial \in T \backslash\{0\}$. So $b f(y)=b^{\prime}$ if $y \in A \backslash\{0\}$. Thus

$$
f(y)=\frac{b^{\prime}}{b} \quad \text { for all } y \in A \backslash\{0\}
$$

Going back to (3.2) we deduce that $b=b^{\prime}$.

If $\alpha \neq 0$, choose $y$ such that $\alpha, y$ are linearly independent in $T^{*}$. Suppose $b \neq b^{\prime}$, then $\alpha+b y, \alpha+b^{\prime} y$ are linearly independent. There exists $\partial \in T^{*}$ such that $\partial(\alpha+b y)=0$ and $\partial\left(\alpha+b^{\prime} y\right) \neq 0$. So from (3.3) we deduce that $f(y)=0$, this is a contradiction. Therefore $b=b^{\prime}$.

(b) From (a) it suffices to show that $\mathscr{A}_{0,0}^{\prime} \not \mathscr{A}_{0,1}^{\prime}$. Set $\mathscr{A}_{0,0}^{\prime}=\bigoplus_{x \in A \backslash\{0\}} F v_{x}, \mathscr{A}_{0,1}^{\prime}=$ $\bigoplus_{x \in A \backslash\{0\}} F v_{x}^{\prime}$ with actions

$$
\begin{aligned}
& \left(t^{x} \partial\right) v_{y}=\partial(y) v_{x+y} \quad \text { for all } \partial \in T, x, y \in A \text { with } y \neq 0 \text { and } x+y \neq 0, \\
& \left(t^{x} \partial\right) v_{y}^{\prime}=\partial(y+x) v_{x+y}^{\prime} \text { for all } \partial \in T, x, y \in A \text { with } y \neq 0 \text { and } x+y \neq 0 .
\end{aligned}
$$

Let $\theta: \mathscr{A}_{0,0}^{\prime} \rightarrow \mathscr{A}_{0,1}^{\prime}$ be an isomorphism. Then

$$
\theta\left(v_{x}\right)=f(x) v_{x}^{\prime} \text { for all } x \in A \backslash\{0\}
$$


where $f(x) \in F^{*}$. From $t^{y} \partial \varphi\left(v_{x}\right)=\varphi\left(\left(t^{y} \partial\right) v_{x}\right)$, it follows that

$$
\partial(x) f(x+y) v_{x+y}^{\prime}=f(x) \partial(x+y) v_{x+y}^{\prime},
$$

to give

$$
\partial(x) f(x+y)=f(x) \partial(x+y), \quad \text { for all } x, y \in A \text { with } x \neq 0 \neq x+y \text {. }
$$

Since $f(x) f(x+y) \neq 0$, we obtain that

$$
\partial(x)=0 \Leftrightarrow \partial(x+y)=0, \quad \forall \partial \in T, x, y \in A \text { with } x \neq 0 \neq x+y .
$$

This is a contradiction since $\operatorname{dim} T \geq 2$. So $\mathscr{A}_{0,0}^{\prime} \not \mathscr{A}_{0,1}^{\prime}$.

Remark. If $\operatorname{dim} T=1$, Proposition 3.2(b) does not hold (see Theorem 2.1).

For any $\alpha \in T^{*}$, we define the $A$-graded $W$-module $\mathscr{A}_{\alpha}(W)=\bigoplus_{x \in A} F v_{x}$ with the actions

$$
\left(t^{x} \partial\right) v_{y}=\partial(y) v_{x+y}, \quad \text { for } y \neq 0 \neq x+y
$$

$$
\begin{aligned}
& \left(t^{x} \partial\right) v_{x}=0, \quad \text { for all } x \in A, \\
& \left(t^{x} \partial\right) v_{0}=\partial(x+\alpha) v_{x}, \quad \text { for all } x \in A \backslash\{0\} ;
\end{aligned}
$$

the $A$-graded $W$-module $\tilde{\mathscr{A}}_{\alpha}(W)=\bigoplus_{x \in A} F v_{x}$ with the actions

$$
\left(t^{x} \partial\right) v_{y}=\partial(y) v_{x+y}, \quad \text { for } y \neq 0 \neq x+y,
$$

$\left(3.4^{\prime}\right) \quad\left(t^{x} \partial\right) v_{x}=0, \quad$ for all $x \in A$,

$$
\left(t^{x} \partial\right) v_{0}=\partial(\alpha) v_{x}, \quad \text { for all } x \in A \backslash\{0\} ;
$$

the $A$-graded $W$-module $\mathscr{B}_{\alpha}(W)=\bigoplus_{x \in A} F v_{x}$ with the actions

$$
\left(t^{x} \partial\right) v_{y}=\partial(x+y) v_{x+y}, \quad \text { for } y \neq 0 \neq x+y,
$$

$$
\left(t^{x} \partial\right) v_{0}=0, \quad \text { for all } x \in A
$$$$
\left(t^{x} \partial\right) v_{x}=\partial(x+\alpha) v_{0}, \quad \text { for all } x \in A \backslash\{0\} ;
$$

the $A$-graded $W$-module $\tilde{\mathscr{B}}_{\alpha}(W)=\bigoplus_{x \in A} F v_{x}$ with the actions 


$$
\left(t^{x} \partial\right) v_{y}=\partial(x+y) v_{x+y}, \quad \text { for } y \neq 0 \neq x+y,
$$

$$
\begin{aligned}
& \left(t^{x} \partial\right) v_{0}=0, \quad \text { for all } x \in A, \\
& \left(t^{x} \partial\right) v_{x}=\partial(\alpha) v_{0}, \quad \text { for all } x \in A \backslash\{0\} .
\end{aligned}
$$

The modules $\mathscr{A}_{\alpha}(W), \tilde{\mathscr{A}}_{\alpha}(W), \mathscr{B}_{\alpha}(W), \tilde{\mathscr{B}}_{\alpha}(W)$ for $\alpha \in T^{*}$ are indecomposable but reducible.

Note that when $\operatorname{dim} T=1$, we have $\tilde{\mathscr{A}}_{\alpha}(W) \simeq \mathscr{A}_{0,1}(W)$, and $\tilde{\mathscr{B}}_{\alpha}(W) \simeq \mathscr{A}_{0,0}(W)$.

Lemma 3.3. Let $W(A, T, \varphi)$ be a simple generalized Witt algebra with $\operatorname{dim} T>1, V=$ $\bigoplus_{x \in A} F v_{x}$ an indecomposable $W$-module with the action

$$
\left(t^{x} \partial\right) v_{y}=\partial(y) v_{x+y} \quad \text { if } y \neq 0 \neq x+y .
$$

Then $V \simeq \mathscr{A}_{0,0}$ or there exists $\alpha \in T^{*}$ such that $V \simeq \mathscr{A}_{\alpha}(W)$, or $V \simeq \tilde{\mathscr{A}}_{\alpha}(W)$ for $\alpha \neq 0$.

Proof. We need only determine $\left(t^{x} \partial\right) v_{y}$ for $x, y \in A$ with $y=0$ or $x+y=0$.

Since $V$ is indecomposable, we know that $T v_{0}=0$, and $\left(t^{x_{0}} \partial_{0}\right) v_{x_{0}} \neq 0$ or $\left(t^{x_{0}} \partial_{0}\right) v_{0} \neq 0$ for some $x_{0} \in A, \partial_{0} \in T$.

Case 1: $\left(t^{x_{0}} \partial_{0}\right) v_{x_{0}} \neq 0$ for some $x_{0} \in A, \partial_{0} \in T$

Suppose $\left(t^{x} \partial\right) v_{x}=f(\partial, x) v_{0}$. Then $f$ is linear in the first variable, and $f(\partial, 0)=$ $f(0, x)=0$. From the equation

$$
\left[t^{x_{1}} \partial_{1}, t^{x_{2}} \partial_{2}\right] v \quad x_{1} \quad x_{2}=t^{x_{1}+x_{2}}\left(\partial_{1}\left(x_{2}\right) \partial_{2}-\partial_{2}\left(x_{1}\right) \partial_{1}\right) v \quad x_{1} x_{2},
$$

we see that

$$
f\left(\partial_{1}\left(x_{2}\right) \partial_{2}-\partial_{2}\left(x_{1}\right) \partial_{1}, x_{1}+x_{2}\right)=-\partial_{2}\left(x_{1}+x_{2}\right) f\left(\partial_{1}, x_{1}\right)+\partial_{1}\left(x_{1}+x_{2}\right) f\left(\partial_{2}, x_{2}\right)
$$

for all $\partial_{1}, \partial_{2} \in T, x_{1}, x_{2} \in A$, even when $x_{1}$ or $x_{2}$ is 0 . If $\partial_{2}=\partial_{1}$ in (3.7), we obtain

$$
\partial_{1}\left(x_{2}-x_{1}\right) f\left(\partial_{1}, x_{1}+x_{2}\right)=\partial_{1}\left(x_{1}+x_{2}\right)\left(f\left(\partial_{1}, x_{2}\right)-f\left(\partial_{1}, x_{1}\right)\right)
$$

for all $x_{1}, x_{2} \in A$. If $\partial_{1}\left(x_{1}\right)=\partial_{1}\left(x_{2}\right) \neq 0,(3.8)$ implies

$$
f\left(\partial_{1}, x_{1}\right)=f\left(\partial_{1}, x_{2}\right) .
$$

If $\partial_{1}\left(x_{1}\right)=0$ and $\partial_{1}\left(x_{2}\right) \neq 0,(3.8)$ implies

$$
f\left(\partial_{1}, x_{1}+x_{2}\right)=f\left(\partial_{1}, x_{2}\right)-f\left(\partial_{1}, x_{1}\right) .
$$

From (3.9) and (3.10) we see that

$$
f\left(\partial_{1}, x_{1}\right)=0, \quad \text { if } \partial_{1}\left(x_{1}\right)=0 .
$$


Claim 1. For any $\partial \in T$, we have $f(\partial, x)=a \partial(x)$ for all $x \in A$ where $a \in F^{*}$ is a constant.

Since $\operatorname{dim} T>1$, we can fix $\partial_{1} \in T$ such that $\operatorname{ker}\left(\partial_{1}\right) \neq 0$. Fix also $x_{1} \in A \backslash\{0\}$ such that $\partial_{1}\left(x_{1}\right) \neq 0$. Choose $x_{2} \in \operatorname{ker}\left(\partial_{1}\right) \backslash\{0\}$. Choose $\partial_{2}^{\prime} \in T$ such that $\partial_{2}^{\prime}\left(x_{2}\right) \neq 0$. Letting $\partial_{2}=\partial_{2}^{\prime}-\partial_{2}^{\prime}\left(x_{1}\right) \partial_{1}\left(x_{1}\right)^{1} \partial_{1}$, then

$$
\partial_{1}\left(x_{1}\right) \neq 0 \neq \partial_{2}\left(x_{2}\right), \quad \partial_{1}\left(x_{2}\right)=\partial_{2}\left(x_{1}\right)=0 \text {. }
$$

From (3.7) we see that

$$
\frac{f\left(\partial_{1}, x_{1}\right)}{\partial_{1}\left(x_{1}\right)}=\frac{f\left(\partial_{2}, x_{2}\right)}{\partial_{2}\left(x_{2}\right)}
$$

Applying (3.11) to (3.12), we deduce that

$$
\frac{f\left(\partial_{1}, x_{1}\right)}{\partial_{1}\left(x_{1}\right)}=\frac{f\left(\partial_{2}^{\prime}, x_{2}\right)}{\partial_{2}^{\prime}\left(x_{2}\right)}
$$

By checking special cases (for example $\partial_{1}\left(x_{1}\right)=0$ ), we see that the condition for (3.13) is only $\partial_{1}\left(x_{2}\right)=0$. Suppose $\partial_{1}\left(x_{3}\right) \neq 0$ for $x_{3} \in A \backslash\{0\}$. Choose $\partial_{3} \in T$ such that $\partial_{3}\left(x_{2}\right) \neq 0$ and $\partial_{3}\left(x_{3}\right)=0$. From $(3.13)$ we see that

$$
\frac{f\left(\partial_{1}, x_{1}\right)}{\partial_{1}\left(x_{1}\right)}=\frac{f\left(\partial_{3}, x_{2}\right)}{\partial_{3}\left(x_{2}\right)}=\frac{f\left(\partial_{2}^{\prime}, x_{3}\right)}{\partial_{2}^{\prime}\left(x_{3}\right)}
$$

Thus

$$
\frac{f\left(\partial_{1}, x_{1}\right)}{\partial_{1}\left(x_{1}\right)}=\frac{f\left(\partial_{2}, x_{2}\right)}{\partial_{2}\left(x_{2}\right)}=a \neq 0, \quad \forall x_{1}, x_{2} \in A, \partial_{1}, \partial_{2} \in T
$$

Claim 1 follows.

Thus, we can choose $v_{0}$ such that

$$
\left(t^{x} \partial\right) v_{x}=-\partial(x) v_{0} \quad \text { for all } x \in A, \partial \in T
$$

Choose $x_{0} \neq 0, \partial_{0}\left(x_{0}\right) \neq 0$. For any $x_{1} \in A, \partial \in T$, from

$$
\begin{aligned}
-\partial_{0}\left(x_{0}\right)\left(t^{x_{1}} \partial_{1}\right) v_{0}+\partial_{1}\left(x_{0}\right) \partial_{0}\left(x_{1}-x_{0}\right) v_{x_{1}} & =\left[t^{x_{1}} \partial_{1}, t^{x_{0}} \partial_{0}\right] v x_{0} \\
=t^{x_{1}+x_{0}}\left(\partial_{1}\left(x_{0}\right) \partial_{0}-\partial_{0}\left(x_{1}\right) \partial_{1}\right) v x_{0} & =\partial_{1}\left(x_{0}\right) \partial_{0}\left(x_{1}-x_{0}\right) v_{x_{1}}
\end{aligned}
$$

we see that $\left(t^{x_{1}} \partial_{1}\right) v_{0}=0$. Thus, $V \simeq \mathscr{A}_{0,0}$.

Case 2: $\left(t^{x_{0}} \partial_{0}\right) v_{0} \neq 0$ for some $x_{0} \in A, \partial_{0} \in T$ and $\left(t^{x} \partial\right) v \quad x=0$ for all $x \in A, \partial \in T$ 
Suppose $\left(t^{x} \partial\right) v_{0}=f(\partial, x) v_{x}$. Then $f$ is linear in the first variable and $f(\partial, 0)=$ $f(0, x)=0$. From $\left[t^{x_{1}} \partial_{1}, t^{x_{2}} \partial_{2}\right] v_{0}=t^{x_{1}+x_{2}}\left(\partial_{1}\left(x_{2}\right) \partial_{2}-\partial_{2}\left(x_{1}\right) \partial_{1}\right) v_{0}$ we see that

$$
f\left(\partial_{2}, x_{2}\right) \partial_{1}\left(x_{2}\right)-f\left(\partial_{1}, x_{1}\right) \partial_{2}\left(x_{1}\right)=f\left(\partial_{1}\left(x_{2}\right) \partial_{2}-\partial_{2}\left(x_{1}\right) \partial_{1}, x_{1}+x_{2}\right)
$$

for all $x_{1}, x_{2} \in A, \partial_{1}, \partial_{2} \in T$ with $x_{1}+x_{2} \neq 0$. By letting $\partial_{2}=\partial_{1}$ in (3.17) we obtain that

$$
\partial_{1}\left(x_{2}\right) f\left(\partial_{1}, x_{2}\right)-\partial_{1}\left(x_{1}\right) f\left(\partial_{1}, x_{1}\right)=\partial_{1}\left(x_{2}-x_{1}\right) f\left(\partial_{1}, x_{1}+x_{2}\right)
$$

for all $x_{1}, x_{2} \in A, \partial_{1} \in T$.

For any nonzero sub-group $A_{1}$ of $A$ with $A_{1} \cap \operatorname{ker}\left(\partial_{1}\right)=0$. Let $W\left(A_{1}, \partial_{1}\right)=$ $\bigoplus_{x \in A_{1}} F t^{x} \partial_{1} \subseteq W(A, T, \varphi), V\left(A_{1}\right)=\bigoplus_{x \in A_{1}} F v_{x}$. Then $W\left(A_{1}, \partial_{1}\right)$ is a centerless generalized Virasoro algebra, and $V\left(A_{1}\right)$ is a module over $W\left(A_{1}, \partial_{1}\right)$. From Theorem 2.2 we know that $V\left(A_{1}\right) \simeq \mathscr{A}_{\alpha}\left(A_{1}\right), V\left(A_{1}\right) \simeq \mathscr{A}_{0,1}\left(A_{1}\right)$, or $V\left(A_{1}\right) \simeq \mathscr{A}_{0,1}^{\prime}\left(A_{1}\right) \oplus F v_{0}$. So there exist $a_{\partial_{1}}, b_{\partial_{1}} \in F$ such that $f\left(\partial_{1}, x\right)=a_{\partial_{1}} \partial_{1}(x)+b_{\partial_{1}}$ for all $x \in A_{1}$. Note that if $V\left(A_{1}\right) \simeq \mathscr{A}_{\alpha}\left(A_{1}\right)$ then $a_{\partial_{1}} \neq 0$, if $V\left(A_{1}\right) \simeq \mathscr{A}_{0,1}\left(A_{1}\right)$ then $a_{\partial_{1}}=0$ and $b_{\partial_{1}} \neq 0$, if $V\left(A_{1}\right) \simeq \mathscr{A}_{0,1}^{\prime}\left(A_{1}\right) \oplus F v_{0}$ then $a_{\partial_{1}}=0$ and $b_{\partial_{1}}=0$.

For $y_{0} \in \operatorname{ker}\left(\partial_{1}\right)$, by replacing $x_{2}$ with $x_{1}+y_{0}$ in (3.18) and $x_{1} \in A_{1}$, we deduce that $f\left(\partial_{1}, x_{1}+y_{0}\right)=f\left(\partial_{1}, x_{1}\right)=a_{\partial_{1}} \partial_{1}\left(x_{1}+y_{0}\right)+b_{\partial_{1}}$, i.e.,

$$
f\left(\partial_{1}, x\right)=a_{\partial_{1}} \partial_{1}(x)+b_{\partial_{1}}, \quad \forall x \in\left(A_{1}+\operatorname{ker}\left(\partial_{1}\right)\right) \backslash \operatorname{ker}\left(\partial_{1}\right) .
$$

For any $y \in A \backslash\left(A_{1}+\operatorname{ker}\left(\partial_{1}\right)\right)$, we fix $y_{0} \in\left(A_{1}+\operatorname{ker}\left(\partial_{1}\right)\right) \backslash \operatorname{ker}\left(\partial_{1}\right)$. If $\left(\mathbb{Z} y+\mathbb{Z} y_{0}\right) \cap$ $\operatorname{ker}\left(\partial_{1}\right)=0$, from (3.19) wee see that there are $a^{\prime}, b^{\prime} \in F$ such that

$$
f\left(\partial_{1}, x\right)=a^{\prime} \partial_{1}(x)+b^{\prime}, \quad \forall x \in\left(\mathbb{Z} y+\mathbb{Z} y_{0}+\operatorname{ker}\left(\partial_{1}\right)\right) \backslash \operatorname{ker}\left(\partial_{1}\right) .
$$

Note that $l y_{0} \notin \operatorname{ker}\left(\partial_{1}\right)$ for $l \neq 0$. By computing $f\left(\partial_{1}, l y_{0}\right), l \in \mathbb{Z}$ from (3.19) and (3.20) we see that $f\left(\partial_{1}, y\right)=a_{\partial_{1}} \partial_{1}(y)+b_{\partial_{1}}$. If $\left(\mathbb{Z} y+\mathbb{Z} y_{0}\right) \cap \operatorname{ker}\left(\partial_{1}\right) \neq 0$, say $m y \in$ $\left(A_{1}+\operatorname{ker}\left(\partial_{1}\right)\right)$ for a positive integer $m$, from (3.19) we see that there are $a^{\prime}, b^{\prime} \in F$ such that

$$
f\left(\partial_{1}, x\right)=a^{\prime} \partial_{1}(x)+b^{\prime}, \quad \forall x \in\left(\mathbb{Z} y+\operatorname{ker}\left(\partial_{1}\right)\right) \backslash \operatorname{ker}\left(\partial_{1}\right)
$$

Note that $l m y \notin \operatorname{ker}\left(\partial_{1}\right)$ for $l \neq 0$. By computing $f\left(\partial_{1}, \operatorname{lm} y\right), l \in \mathbb{Z}$ from (3.20) and (3.21) we see that $f\left(\partial_{1}, y\right)=a_{\partial_{1}} \partial_{1}(y)+b_{\partial_{1}}$. Thus

$$
f\left(\partial_{1}, x\right)=a_{\partial_{1}} \partial_{1}(x)+b_{\partial_{1}}, \quad \forall x \in A \backslash \operatorname{ker}\left(\partial_{1}\right) .
$$

Since $f(\partial, x)$ is linear in the first variable, we see that

$$
a_{\partial_{1}+\partial_{2}}\left(\partial_{1}(x)+\partial_{2}(x)\right)+b_{\partial_{1}+\partial_{2}}=a_{\partial_{1}} \partial_{1}(x)+b_{\partial_{1}}+a_{\partial_{2}} \partial_{2}(x)+b_{\partial_{2}},
$$


which implies that

$$
a_{\hat{\partial}_{1}+\partial_{2}}=a_{\partial_{1}}=a_{\partial_{2}}, \quad \forall \partial_{1}, \partial_{2} \in T,
$$

and that $b_{\partial}$ is linear in $\partial \in T$, which ensures that there exists an $\alpha \in T^{*}$ such that $b_{\partial}=$ $\alpha(\partial), \forall \partial \in T$. So

$$
f(\partial, x)=a \partial(x)+\alpha(\partial), \quad \forall \partial \in T, x \in A \backslash \operatorname{ker}(\partial),
$$

where $a \in F, \alpha \in T^{*}$. Since $f(\partial, x)$ is linear in $\partial$, we deduce that

$$
f(\partial, x)=a \partial(x)+\alpha(\partial), \quad \forall \partial \in T, x \in A \backslash\{0\},
$$

We see that $V \simeq \mathscr{A}_{a{ }^{1}{ }_{\alpha}}(W)$ if $a \neq 0, V \simeq \tilde{\mathscr{A}}_{\alpha}(W)$ if $a=0$.

Lemma 3.4. Let $W(A, T, \varphi)$ be a simple generalized Witt algebra with $\operatorname{dim} T>1, V=$ $\bigoplus_{x \in A} F v_{x}$ an indecomposable $W$-module with the action

$$
\left(t^{x} \partial\right) v_{y}=\partial(x+y) v_{x+y}, \quad \text { for all } y \neq 0 \neq x+y .
$$

Then $V \simeq \mathscr{A}_{0,1}$ or there exists $\alpha \in T^{*}$ such that $V \simeq \mathscr{B}_{\alpha}(W)$, or $V \simeq \tilde{\mathscr{B}}_{\alpha}(W)$ for $\alpha \neq 0$.

The proof is similar to that of Lemma 3.3. We omit the details.

From the above two lemmas, we can easily see

Lemma 3.5. Let $W=W(A, T, \varphi)$ be a simple generalized Witt algebra with $\operatorname{dim} T>1$. Assume $V=\bigoplus_{x \in A} V_{x+\alpha}$ is a $W$-module with $\operatorname{dim} V_{\alpha+x} \leq 1$ for all $x \in A$, where $\alpha \in T^{*}$, $V_{x+\alpha}=\{v \in V \mid \partial v=\partial(x+\alpha) v \forall \partial \in T\}$. Let $v_{x} \in V_{\alpha+x}$ (possibly $v_{x}=0$ if $\alpha+x=0$ ), and

$$
\left(t^{x} \partial\right) v_{y}=\partial(y+\alpha+b x) v_{x+y} \quad \text { for all } x, y \in A, \partial \in T
$$

with $b \in F, y+\alpha \neq 0 \neq x+y+\alpha$. Then $V$ is isomorphic to one of the following for appropriate $b \in F$ and $\beta \in T^{*}: F v_{0}, \mathscr{A}_{\alpha, b}(W), \mathscr{A}_{\beta}(W), \tilde{\mathscr{A}}_{\beta}(W), \mathscr{B}_{\beta}(W), \tilde{\mathscr{A}}_{\beta}(W)$, $\mathscr{A}_{0, b}^{\prime}(W)(b \in\{0,1\}), \mathscr{A}_{0, b}^{\prime}(W) \oplus F v_{0}(b \in\{0,1\})$.

Similar to Theorem 2.1, we have

Theorem 3.6. Let $W=W(A, T, \varphi)$ be a simple generalized Witt algebra with $\operatorname{dim} T>1$. Among the $W$-modules $\mathscr{A}_{\alpha, b}(W), \mathscr{A}_{\alpha}(W), \mathscr{B}_{\alpha}(W)$ for $\alpha \in T^{*}, b \in F$, and their nontrivial sub-quotients, we have only the following module isomorphisms:

(i) $\mathscr{A}_{\alpha, b}(W) \simeq \mathscr{A}_{\alpha^{\prime}, b}(W)$ if $a-a^{\prime} \in M$,

(ii) $\mathscr{A}_{a, b}^{\prime}(W) \simeq \mathscr{A}_{a^{\prime}, b}^{\prime}(W)$ if $a-a^{\prime} \in M$,

(iii) $\mathscr{A}_{\alpha}^{\prime}(W) \simeq \tilde{\mathscr{A}}_{\alpha}^{\prime}(W) \simeq \mathscr{A}_{0,0}^{\prime}(W)$,

(iv) $\quad \mathscr{B}_{\alpha}^{\prime}(W) \simeq \tilde{\mathscr{B}}_{\alpha}^{\prime}(W) \simeq \mathscr{A}_{0,1}^{\prime}(W)$. 


\section{$\S 4$ Classification of modules with weight multiplicity 1}

Now we are ready to show the main classification theorem over an algebraically closed field.

Theorem 4.1. Let $W=W(A, T, \varphi)$ be a simple generalized Witt algebra over an algebraically closed field $F$ of characteristic 0 . Assume $V=\bigoplus_{x \in A} V_{\alpha+x}$ is a $W$-module with $\operatorname{dim} V_{\alpha+x} \leq 1$ for all $x \in A$, where $\alpha \in T^{*}$ and

$$
V_{\alpha+x}=\{v \in V \mid \partial v=\partial(\alpha+x) v, \text { for all } \partial \in T\} .
$$

Then $V$ is isomorphic to one of the following modules for appropriate $b \in F$ and $\beta \in T^{*}$ :

(i) the trivial module $\mathrm{Fv}_{0}$,

(ii) $\mathscr{A}_{\alpha, b}(W)$,

(iii) $\mathscr{A}_{\beta}(W), \tilde{\mathscr{A}}_{\beta}(W)$,

(iv) $\mathscr{B}_{\beta}(W), \tilde{\mathscr{B}}_{\beta}(W)$,

(v) $\mathscr{A}_{0, b}^{\prime}(W)$, where $b \in\{0,1\}$,

(vi) $\mathscr{A}_{0, b}^{\prime} \oplus F v_{0}$ (direct sum of modules), where $b \in\{0,1\}$.

Proof. If $\alpha \in A$, we always choose $\alpha=0$. If $\operatorname{dim} V=1, V$ is a trivial $W$-module which is (i). If $\operatorname{dim} T=1$, Theorem 2.2 ensures the theorem.

Next we suppose $\operatorname{dim} V>1$, and $\operatorname{dim} T \geq 2$. If $B$ is a sub-group of $A, T_{0}$ is a subspace of $T$ such that $\left.\varphi\right|_{T_{0} \times B}$ is non-degenerate, then $W\left(B, T_{0}, \varphi\right)$ is also a simple Witt algebra, and $V(\beta, B)=\bigoplus_{x \in B} V_{\beta+x}$ is a $W\left(B, T_{0}, \varphi\right)$-module.

Claim 1. $V_{\alpha+x} \neq 0$ if $\alpha+x \neq 0$.

Otherwise suppose $x_{0} \in A$ such that $V_{\alpha+x_{0}}=0$, and $\alpha+x_{0} \neq 0$. Since $\operatorname{dim} V>1$, we can choose $x_{1} \in A$ such that $V_{\alpha+x_{1}} \neq 0, \alpha+x_{1} \neq 0$. Choose $\partial_{0} \in T$ such that $\partial_{0}\left(\alpha+x_{0}\right) \neq 0$, and $\partial_{0}\left(x_{1}-x_{0}\right) \neq 0$. Then $W\left(\mathbb{Z}\left(x_{1}-x_{0}\right), F \partial_{0}, \varphi\right)$ is a centerless Virasoro algebra, and

$$
\left.V\left(\alpha+x_{0}, Z\left(x_{1}+x_{0}\right)\right)=\bigoplus_{k \in Z} V_{\alpha+x_{0}+k\left(x_{1}\right.} x_{0}\right)
$$

is a nontrivial $W\left(\mathbb{Z}\left(x_{1}-x_{0}\right), F \partial_{0}, \varphi\right)$-module with one weight missing. So

$$
V\left(\alpha+x_{0}, \mathbb{Z}\left(x_{1}-x_{0}\right)\right) \simeq \mathscr{A}_{0,0}^{\prime}\left(W\left(\mathbb{Z}\left(x_{1}-x_{0}\right), F \partial_{0}, \varphi\right)\right) .
$$

This forces $\partial_{0}(\alpha+x)=0$, which is a contradiction. So Claim 1 follows.

Claim 2. If $\operatorname{dim} T=2$, and $A \simeq \mathbb{Z} \oplus \mathbb{Z}$, then the statement in Theorem 4.1 holds. 
Since $F$ is algebraically closed, we can choose a basis $\partial_{0}, \partial_{1}$ for $T$ such that $\operatorname{ker} \partial_{0}=$ ker $\partial_{1}=0$. Because $W\left(A, F \partial_{0}, \varphi\right)$ is a centerless Virasoro algebra, from Theorem 2.2 there exists $b_{0}$ such that we can choose $v_{y} \in V_{\alpha+y}$ for all $y \in A \backslash\{0\}$ satisfying:

$$
\left(t^{x} \partial_{0}\right) v_{y}=\partial_{0}\left(y+\alpha+b_{0} x\right) v_{x+y}, \quad \forall x, y \text { with } y+\alpha \neq 0 \neq \alpha+x+y .
$$

Let $f(x, y) \in F$ be such that

$$
\left(t^{x} \partial_{1}\right) v_{y}=\left(\partial_{1}\left(y+\alpha+b_{0} x\right)+f(x, y)\right) v_{x+y}, \quad \forall x, y \text { with } y+\alpha \neq 0 \neq x+y+\alpha .
$$

We are going to prove that $f(x, y)=0$ for all $x, y$ with $y+\alpha \neq 0 \neq \alpha+x+y$.

From

$$
\begin{aligned}
& {\left[t^{x_{1}} \partial_{1}, t^{x_{2}} \partial_{1}\right] v_{y}} \\
& =\partial_{1}\left(x_{2}-x_{1}\right)\left(t^{x_{1}+x_{2}} \partial_{1}\right) v_{y} \\
& =\partial_{1}\left(x_{2}-x_{1}\right)\left(\partial_{1}\left(y+\alpha+b_{0}\left(x_{1}+x_{2}\right)\right)+f\left(x_{1}+x_{2}, y\right)\right) v_{x_{1}+x_{2}+y}, \\
& {\left[t^{x_{1}} \partial_{1}, t^{x_{2}} \partial_{1}\right] v_{y}} \\
& =\left[\left(\partial_{1}\left(y+\alpha+b_{0} x_{2}\right)+f\left(x_{2}, y\right)\right)\left(\partial_{1}\left(y+x_{2}+\alpha+b_{0} x_{1}\right)+f\left(x_{1}, x_{2}+y\right)\right)\right. \\
& \quad-\left(\partial_{1}\left(y+\alpha+b_{0} x_{1}\right)+f\left(x_{1}, y\right)\right)\left(\partial_{1}\left(y+x_{1}+\alpha+b_{0} x_{2}\right)\right. \\
& \left.\left.\quad+f\left(x_{2}, x_{1}+y\right)\right)\right] v_{x_{1}+x_{2}+y},
\end{aligned}
$$

we obtain

$$
\begin{aligned}
& \partial_{1}\left(x_{2}-x_{1}\right) f\left(x_{1}+x_{2}, y\right) \\
& =\partial_{1}\left(y+\alpha+b_{0} x_{2}\right) f\left(x_{1}, x_{2}+y\right) \\
& \quad+\partial_{1}\left(y+x_{2}+\alpha+b_{0} x_{1}\right) f\left(x_{2}, y\right)+f\left(x_{2}, y\right) f\left(x_{1}, x_{2}+y\right) \\
& \quad-\partial_{1}\left(y+\alpha+b_{0} x_{1}\right) f\left(x_{2}, x_{1}+y\right)-f\left(x_{1}, y\right) f\left(x_{2}, x_{1}+y\right) \\
& \quad-\partial_{1}\left(y+x_{1}+\alpha+b_{0} x_{2}\right) f\left(x_{1}, y\right)
\end{aligned}
$$

for all $x_{1}, x_{2}, y \in A$ with $y+\alpha \neq 0 \neq y+x_{1}+x_{2}+\alpha$, and $y+x_{1}+\alpha \neq 0 \neq$ $y+x_{2}+\alpha$.

From the computation

$$
\begin{aligned}
{\left[t^{x_{1}} \partial_{0}, t^{x_{2}} \partial_{1}\right] v_{y}=} & t^{x_{1}+x_{2}}\left(\partial_{0}\left(x_{2}\right) \partial_{1}-\partial_{1}\left(x_{1}\right) \partial_{0}\right) v_{y} \\
=\left[\partial _ { 0 } ( x _ { 2 } ) \left(\partial_{1}(y+\alpha+\right.\right. & \left.\left.b_{0}\left(x_{1}+x_{2}\right)\right)+f\left(x_{1}+x_{2}, y\right)\right) \\
& \left.\quad-\partial_{1}\left(x_{1}\right) \partial_{0}\left(y+\alpha+b_{0}\left(x_{1}+x_{2}\right)\right)\right] v_{x_{1}+x_{2}+y}
\end{aligned}
$$




$$
\begin{aligned}
& {\left[t^{x_{1}} \partial_{0}, t^{x_{2}} \partial_{1}\right] v_{y}} \\
& =\left[( \partial _ { 1 } ( y + \alpha + b _ { 0 } x _ { 2 } ) + f ( x _ { 2 } , y ) ) \left(\partial_{0}\left(x_{2}+y+\alpha+b_{0} x_{1}\right)\right.\right. \\
& \left.-\partial_{0}\left(y+\alpha+b_{0} x_{1}\right)\left(\partial_{1}\left(x_{1}+y+\alpha+b_{0} x_{2}\right)+f\left(x_{2}, x_{1}+y\right)\right)\right] v_{x_{1}+x_{2}+y},
\end{aligned}
$$

we obtain

$$
\begin{aligned}
& \partial_{0}\left(x_{2}\right) f\left(x_{1}+x_{2}, y\right) \\
& =\partial_{0}\left(x_{2}+y+\alpha+b_{0} x_{1}\right) f\left(x_{2}, y\right)-\partial_{0}\left(y+\alpha+b_{0} x_{1}\right) f\left(x_{2}, x_{1}+y\right),
\end{aligned}
$$

for all $x_{1}, x_{2}, y \in A$ with $x_{1}+y+\alpha \neq 0 \neq x_{2}+y+\alpha, y+\alpha \neq 0 \neq x_{1}+x_{2}+y+\alpha$. From (4.5) it follows that

$$
f(0, y)=0 .
$$

By setting $x_{2}=-x_{1}$, in (4.7) and using (4.8) we obtain

$$
\partial_{0}\left(y+\alpha+\left(b_{0}-1\right) x_{1}\right) f\left(-x_{1}, y\right)=\partial_{0}\left(y+\alpha+b_{0} x_{1}\right) f\left(-x_{1}, y+x_{1}\right),
$$

$\forall x_{1}, y \in A$ with $y+\alpha \pm x_{1} \neq 0 \neq y+\alpha$. Replacing $y$ with $y+k x_{1}$ in (4.9) gives

$$
\begin{aligned}
& \partial_{0}\left(y+\alpha+\left(b_{0}+k-1\right) x_{1}\right) f\left(-x_{1}, y+k x_{1}\right) \\
& =\partial_{0}\left(y+\alpha+\left(b_{0}+k\right) x_{1}\right) f\left(-x_{1}, y+(k+1) x_{1}\right),
\end{aligned}
$$

for all $x, y \in A$ with $y+\alpha+(k \pm 1) x_{1} \neq 0 \neq y+\alpha+k x_{1}$.

For any fixed pair $\left(x_{1}, y\right)$, actually (4.10) holds for all $k \in \mathbb{Z}$ except for at most three $k$, say $k=k_{0}, k_{0}-1, k_{0}-2$. If such a $k_{0}$ does not exists we should ignore all restrictions related to $k_{0}$. Noting that the right hand side of (4.10) is the left hand side with $k$ replaced by $k+1$, so there exist $a_{x, y} \in F$ such that

$$
f\left(-x_{1}, y+k x_{1}\right)=\frac{a_{x_{1}, y}}{\partial_{0}\left(y+\alpha+\left(b_{0}+k-1\right) x_{1}\right)},
$$

i.e.

$$
f\left(x_{1}, y+k x_{1}\right)=\frac{a_{x_{1}, y}}{\partial_{0}\left(y+\alpha+\left(k+1-b_{0}\right) x_{1}\right)},
$$

for all $k>k_{0}$ (or $k<k_{0}-2$ ), where $a_{x_{1}, y} \in F$ also depends on $y$. Note that for $k>k_{0}$ and $k<k_{0}-2, a_{x_{1}, y}$ is maybe different. But there will be no conflict we use the same notation. Setting $x_{1}=k x_{2}, y=y+j x_{2}$ in (4.7) and using (4.11), we deduce that 


$$
\begin{aligned}
\partial_{0}\left(x_{2}\right) f\left((k+1) x_{2}, y+j x_{2}\right) \\
=\partial_{0}\left(y+\alpha+\left(k b_{0}+1+j\right) x_{2}\right) f\left(x_{2}, y+j x_{2}\right) \\
\quad-\partial_{0}\left(y+\alpha+\left(k b_{0}+j\right) x_{2}\right) f\left(x_{2}, y+(k+j) x_{2}\right) \\
=a_{x_{2}, y}\left(\frac{\partial_{0}\left(y+\alpha+\left(k b_{0}+1+j\right) x_{2}\right)}{\partial_{0}\left(y+\alpha+\left(j+1-b_{0}\right) x_{2}\right)}-\frac{\partial_{0}\left(y+\alpha+\left(k b_{0}+j\right) x_{2}\right)}{\partial_{0}\left(y+\alpha+\left(k+j+1-b_{0}\right) x_{2}\right)}\right) \\
=\frac{a_{x_{2}, y}(k+1) \partial_{0}\left(x_{2}\right) \partial_{0}\left(y+\alpha+\left(j+1+(k-1) b_{0}\right) x_{2}\right)}{\partial_{0}\left(y+\alpha+\left(j+1-b_{0}\right) x_{2}\right) \partial_{0}\left(y+\alpha+\left(k+j+1-b_{0}\right) x_{2}\right)} .
\end{aligned}
$$

So

$$
f\left(k x_{2}, y+j x_{2}\right)=\frac{k a_{x_{2}, y} \partial_{0}\left(y+\alpha+\left(j+1+(k-2) b_{0}\right) x_{2}\right)}{\partial_{0}\left(y+\alpha+\left(j+1-b_{0}\right) x_{2}\right) \partial_{0}\left(y+\alpha+\left(k+j-b_{0}\right) x_{2}\right)},
$$

i.e.,

(4.12) $f\left(j x_{2}, y+k x_{2}\right)=\frac{j a_{x_{2}, y} \partial_{0}\left(y+\alpha+\left(k+1+(j-2) b_{0}\right) x_{2}\right)}{\partial_{0}\left(y+\alpha+\left(k+1-b_{0}\right) x_{2}\right) \partial_{0}\left(y+\alpha+\left(j+k-b_{0}\right) x_{2}\right)}$,

for all $j>k_{0}, k>k_{0}$ (or $\left.j<k_{0}-2, k<k_{0}-2\right)$. Thus,

$$
\begin{aligned}
& f\left(j x_{1}, y+k j x_{1}\right)=\frac{a_{j x_{1}, y}}{\partial_{0}\left(y+\alpha+\left(k+1-b_{0}\right) j x_{1}\right)} \\
& =\frac{j a_{x_{1}, y} \partial_{0}\left(y+\alpha+\left(k j+1+(j-2) b_{0}\right) x_{1}\right)}{\partial_{0}\left(y+\alpha+\left(k j+1-b_{0}\right) x_{1}\right) \partial_{0}\left(y+\alpha+\left(k+k j-b_{0}\right) x_{1}\right)},
\end{aligned}
$$

for all $j>k_{0}, k>k_{0}$ (or $\left.j<k_{0}-2, k<k_{0}-2\right)$. So

$$
\begin{aligned}
& a_{j x_{1}, y} \partial_{0}\left(y+\alpha+\left(k j+1-b_{0}\right) x_{1}\right) \partial_{0}\left(y+\alpha+\left(k+k j-b_{0}\right) x_{1}\right) \\
& =j a_{x_{1}, y} \partial_{0}\left(y+\alpha+\left(k+1-b_{0}\right) j x_{1}\right) \partial_{0}\left(y+\alpha+\left(k j+1+(j-2) b_{0}\right) x_{1}\right),
\end{aligned}
$$

for all $j>k_{0}, k>k_{0}$ (or $j<k_{0}-2, k<k_{0}-2$ ). Comparing the coefficients of $k^{2}$, we see that

$$
a_{j x_{1}, y} j \partial_{0}\left(x_{1}\right)(1+j) \partial_{0}\left(x_{1}\right)=j a_{x_{1}, y} j \partial_{0}\left(x_{1}\right) j \partial_{0}\left(x_{1}\right),
$$

i.e.,

(4.14) $\quad a_{j x_{1}, y}=\frac{j^{2}}{j+1} a_{x_{1}, y}, \quad \forall j>k_{0}\left(\right.$ or $\left.j<k_{0}-2\right)$. 
Applying (4.14) to (4.13) gives

$$
\begin{aligned}
& j \partial_{0}\left(y+\alpha+\left(k j+1-b_{0}\right) x_{1}\right) \partial_{0}\left(y+\alpha+\left(k+k j-b_{0}\right) x_{1}\right) a_{x, y} \\
& =(j+1) \partial_{0}\left(y+\alpha+\left(k+1-b_{0}\right) j x_{1}\right) \partial_{0}\left(y+\alpha+\left(k j+1+(j-2) b_{0}\right) x_{1}\right) a_{x_{1}, y}
\end{aligned}
$$

for all $j, k \in Z$ with $j>k_{0}, k>k_{0}$ (or $j<k_{0}-2, k<k_{0}-2$ ).

It is clear that (4.15) is a polynomial identity in $j$ and $k$, so (4.15) holds for all $j, k \in F$. Setting $j=0,-1$ in (4.15) respectively, we obtain

$$
\begin{aligned}
& a_{x_{1}, y} \partial_{0}(y+\alpha) \partial_{0}\left(y+\alpha+\left(1-2 b_{0}\right) x_{1}\right)=0 \\
& a_{x_{1}, y} \partial_{0}\left(y+\alpha+\left(1-k-b_{0}\right) x_{1}\right) \partial_{0}\left(y+\alpha-b_{0} x_{1}\right)=0, \quad \forall k \in \mathbb{Z}
\end{aligned}
$$

From (4.17) we get

$$
a_{x_{1}, y} \partial_{0}\left(y+\alpha-b_{0} x_{1}\right)=0, \quad \text { if } x_{0} \neq 0
$$

Applying this to (4.16), we see that

$$
a_{x_{1}, y}=0, \quad b=0 \quad \text { or } \quad b=1
$$

Combining this with (4.18) yields one of the following

(i) $a_{x_{1}, y} \neq 0, b_{0}=1$ and $y+\alpha=x_{1} \neq 0$,

(ii) $a_{x_{1}, y} \neq 0, y+\alpha=0$ and $b_{0}=0$,

(iii) $a_{x_{1}, y}=0$.

In the first case, we deduce that $y=x_{1}$ (we have chosen $\alpha=0$ ). In the second case, we deduce that $y=0$ (we have chosen $\alpha=0$ ). Thus $a_{x, y}=0$ for all $x, y \in A$ with $x \neq y, y+\alpha \neq 0 \neq x+y+\alpha$. From (4.11) we see that $f(x, y)=0$ for all $x, y \in A$ with $y+\alpha \neq 0 \neq x+y+\alpha$. Therefore from this fact and Lemma 3.5, we deduce that $V \simeq \mathscr{A}_{\alpha, b}(W), \mathscr{A}_{0,0}^{\prime}(W), \mathscr{A}_{0,1}^{\prime}(W), \mathscr{A}_{\beta}(W), \tilde{\mathscr{A}}_{\beta}(W), \mathscr{B}_{\beta}(W), \tilde{\mathscr{B}}_{\beta}(W)$, $\mathscr{A}_{0,0}^{\prime}(W) \oplus F v_{0}, \mathscr{A}_{0,1}^{\prime}(W) \oplus F v_{0}$, for some $\beta \in T^{*}, b \in F$. Claim 2 follows.

Claim 3. If $\operatorname{dim} T=2$, then the statement in Theorem 4.1 holds.

We say that a sub-group $B$ of $A$ is non-degenerate if $\left.\varphi\right|_{T \times B}$ is non-degenerate. For any sub-group $B$ of $A$, recall that

$$
V\left(\alpha+x_{0}, B\right)=\bigoplus_{x \in B} V_{\alpha+x_{0}+x}
$$

From Zorn's Lemma and Claim 2, there exists a maximal non-degenerate sub-group $M_{0}$ of $A$ satisfying the following condition: 
(C1) Let $\left\{x_{i} \mid i \in I\right\}$ be the set of all representatives of cosets of $M_{0}$ in $A$ with $0 \in I$, $x_{0}=0$. For any $i \in I$,

$$
V\left(\alpha+x_{i}, M_{0}\right) \simeq \begin{cases}\mathscr{A}_{\alpha+x_{i}, b_{i}^{\prime}}\left(W\left(M_{0}, T, \varphi\right)\right), & \\ \mathscr{A}_{\beta}\left(W\left(M_{0}, T, \varphi\right)\right), & b_{0}^{\prime}=0, \\ \tilde{A}_{\beta}\left(W\left(M_{0}, T, \varphi\right)\right), & b_{0}^{\prime}=0, \\ \mathscr{B}_{\beta}\left(W\left(M_{0}, T, \varphi\right)\right), & b_{0}^{\prime}=1, \\ \tilde{\mathscr{B}}_{\beta}\left(W\left(M_{0}, T, \varphi\right)\right), & b_{0}^{\prime}=1, \\ \mathscr{A}_{0, b_{0}^{\prime}}^{\prime}\left(W\left(M_{0}, T, \varphi\right)\right), & b_{0}^{\prime} \in\{0,1\}, \\ \mathscr{A}_{0, b_{0}^{\prime}}^{\prime}\left(W\left(M_{0}, T, \varphi\right)\right) \oplus F v_{0}, & b_{0}^{\prime} \in\{0,1\},\end{cases}
$$

for some $\beta \in T^{*}, b_{i}^{\prime} \in F$. Note that the last six cases maybe occur only when $i=0$ and $\alpha+x_{i}=0$, and we regard $b_{0}^{\prime}=0$ for $\mathscr{A}_{\beta}$ and $\tilde{\mathscr{A}}_{\beta}, b_{0}^{\prime}=1$ for $\mathscr{B}_{\beta}$ and $\tilde{\mathscr{B}}_{\beta}$, and hereinafter.

To prove Claim 3 it suffices to show that $A=M_{0}$. Otherwise suppose $M_{0} \neq A$. So $|I|>1$, and $x_{1} \in A \backslash M_{0}$ for $1 \in I \backslash\{0\}$. Let $M_{1}=M_{0}+\mathbb{Z} x_{1},\left\{y_{i} \mid j \in J\right\}$ be the set of all representatives of cosets of $M_{1}$ in $A$, with $0 \in J$ and $y_{0}=0$. Let $\left\{i x_{1} \mid i \in K\right\}$ be the set of all representatives of cosets of $M_{0}$ in $M_{1}$, where $K \subseteq \mathbb{Z}$ and $|K|>1$. Fix an arbitrary $x_{1}^{\prime} \in x_{1}+M_{0}$.

Now we fix $y_{j}$, from $(\mathrm{C} 1)$ we see that

$$
V\left(\alpha+y_{j}+i x_{1}^{\prime}, M_{0}\right) \simeq \begin{cases}\mathscr{A}_{\alpha+y_{j}+i x_{1}^{\prime}, b_{i}}\left(W\left(M_{0}, T, \varphi\right)\right), & \\ \mathscr{A}_{\beta}\left(W\left(M_{0}, T, \varphi\right)\right), & b_{0}=0, \\ \tilde{\mathscr{A}}_{\beta}\left(W\left(M_{0}, T, \varphi\right)\right), & b_{0}=0, \\ \mathscr{B}_{\beta}\left(W\left(M_{0}, T, \varphi\right)\right), & b_{0}=1, \\ \tilde{\mathscr{B}}_{\beta}\left(W\left(M_{0}, T, \varphi\right)\right), & b_{0}=1, \\ \mathscr{A}_{0, b_{0}}^{\prime}\left(W\left(M_{0}, T, \varphi\right)\right), & b_{0} \in\{0,1\}, \\ \mathscr{A}_{0, b_{0}}^{\prime}\left(W\left(M_{0}, T, \varphi\right)\right) \oplus F v_{0}, & b_{0} \in\{0,1\},\end{cases}
$$

for all $i \in K$, where $\beta \in T^{*}, b_{i} \in F$. Note that if $i \in K \backslash\{0\}$, in (4.22) only the first case occurs, since we have assumed that $\alpha+y_{j}+i x_{1}^{\prime} \notin M_{0}$ if $i \neq 0$. For this fixed $y_{j}$, for any $z_{0} \in M_{0} \backslash\{0\}$ with the property that $N_{0}=\mathbb{Z} z_{0}+\mathbb{Z} x_{1}^{\prime}$ is non-degenerate, choose $\partial_{0} \in T$ such that and $\partial_{0}\left(z_{0}\right) \neq 0$. Fix any $z_{1} \in M_{0}$.

From Claim 2 we know that there exists $b \in F$ such that

$$
V\left(\alpha+z_{1}+y_{j}, N_{0}\right) \simeq \begin{cases}\mathscr{A}_{\alpha+z_{1}+y_{j}, b}\left(W\left(N_{0}, T, \varphi\right)\right), & \\ \mathscr{A}_{\beta^{\prime}}\left(W\left(N_{0}, T, \varphi\right)\right), & b=0, \\ \tilde{\mathscr{A}}_{\beta^{\prime}}\left(W\left(N_{0}, T, \varphi\right)\right), & b=0, \\ \mathscr{B}_{\beta^{\prime}}\left(W\left(N_{0}, T, \varphi\right)\right), & b=1, \\ \tilde{\mathscr{B}}_{\beta^{\prime}}\left(W\left(N_{0}, T, \varphi\right)\right), & b=1, \\ \mathscr{A}_{0, b}^{\prime}\left(W\left(N_{0}, T, \varphi\right)\right), & b \in\{0,1\}, \\ \mathscr{A}_{0, b}^{\prime}\left(W\left(N_{0}, T, \varphi\right)\right) \oplus F v_{0}, & b \in\{0,1\},\end{cases}
$$


for some $\beta^{\prime} \in T^{*}$. The last six cases possibly occur if $i=j=0, z_{1}+\alpha+y_{j} \in N_{0}$. As modules over the Virasoro algebra $W_{0,0}=W\left(\mathbb{Z} z_{0}, F \partial_{0}, \varphi\right)$, from (4.22) we deduce

$$
V\left(\alpha+z_{1}+y_{j}+i x_{1}^{\prime}, \mathbb{Z} z_{0}\right) \simeq \begin{cases}\mathscr{A}_{\alpha+z_{1}+y_{j}+i x_{i}^{\prime}, b_{i}}\left(W_{0,0}\right), & \\ \mathscr{A}_{\beta}\left(W_{0,0}\right), & b_{0}=0, \\ \tilde{\mathscr{A}}_{\beta}\left(W_{0,0}\right), & b_{0}=0, \\ \mathscr{B}_{\beta}\left(W_{0,0}\right), & b_{0}=1, \\ \tilde{\mathscr{B}}_{\beta}\left(W_{0,0}\right), & b_{0}=1, \\ \mathscr{A}_{0, b_{0}}^{\prime}\left(W_{0,0}\right), & b_{0} \in\{0,1\}, \\ \mathscr{A}_{0, b_{0}}^{\prime}\left(W_{0,0}\right) \oplus F v_{0}, & b_{0} \in\{0,1\} .\end{cases}
$$

The last six cases possibly occur if $i=j=0, z_{1}+\alpha+y_{j}+i x_{i}^{\prime} \in \mathbb{Z} z_{0}$. From (4.23) we see that

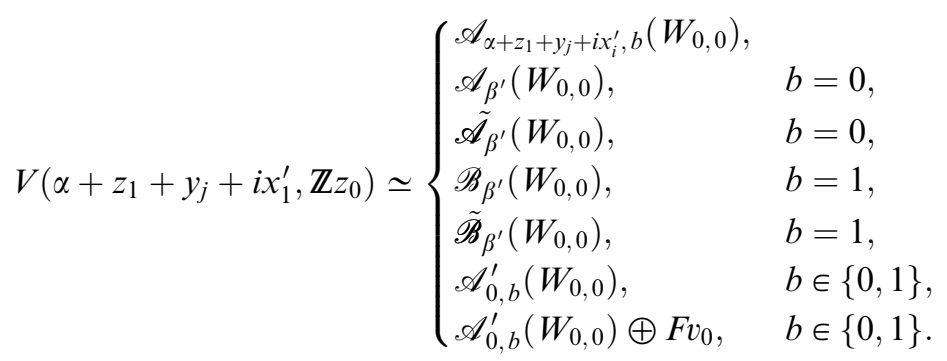

From Theorem 2.2 we see that $\beta=\beta^{\prime}$.

Claim 3.1. In (4.22) and (4.23), we have $b=b_{i}$ for all $i \in K$ correspondingly.

Suppose $b \neq b_{i}$ for some $i \in K$, say, $b \neq b_{1}$. From (4.24), (4.25) and Theorem 2.1, we see that $b, b_{1} \in\{0,1\}$. Without loss of generality we assume that $b=1, b_{1}=0$. From (4.22) and (4.23) we can choose $v_{y_{j}+z_{1}+k z_{0}+x_{1}^{\prime}}, v_{y_{j}+z_{1}+k z_{0}+x_{1}^{\prime}}^{\prime} \in V_{y_{j}+z_{1}+\alpha+k z_{0}}$ for all $k \in \mathbb{Z}$ such that, for all $k_{1}, k_{2}, k \in \mathbb{Z}$ and $\partial \in T$,

$$
v_{y_{j}+z_{1}+k z_{0}+x_{1}^{\prime}}=f(k) v_{y_{j}+z_{1}+k z_{0}+x_{1}^{\prime}}^{\prime}, \quad f(k) \neq 0,
$$

$$
\begin{aligned}
& \left(t^{k_{1} z_{0}} \partial\right) v_{y_{j}+z_{1}+k_{2} z_{0}+x_{1}^{\prime}}=\partial\left(y_{j}+z_{1}+\alpha+x_{1}^{\prime}+k_{2} z_{0}\right) v_{y_{j}+z_{1}+\left(k_{1}+k_{2}\right) z_{0}+x_{1}^{\prime}}, \\
& \left(t^{k_{1} z_{0}} \partial\right) v_{y_{j}+z_{1}+k_{2} z_{0}+x_{1}^{\prime}}^{\prime}=\partial\left(y_{j}+z_{1}+\alpha+x_{1}^{\prime}+\left(k_{1}+k_{2}\right) z_{0}\right) v_{y_{j}+z_{1}+\left(k_{1}+k_{2}\right) z_{0}+x_{1}^{\prime}}^{\prime} .
\end{aligned}
$$

Thus, we obtain that

$$
f\left(k_{2}\right) \partial\left(y_{j}+z_{1}+\alpha+x_{1}^{\prime}+\left(k_{1}+k_{2}\right) z_{0}\right)=f\left(k_{1}+k_{2}\right) \partial\left(y_{j}+z_{1}+\alpha+x_{i}^{\prime}+k_{2} z_{0}\right),
$$

for all $k_{1}, k_{2}, k \in \mathbb{Z}, \partial \in T$. Letting $k_{2}=0$ in (4.28) yields $f(0) \partial\left(y_{j}+z_{1}+\alpha+x_{1}^{\prime}+k_{1} z_{0}\right)=f\left(k_{1}\right) \partial\left(y_{j}+z_{1}+\alpha+x_{1}^{\prime}\right), \quad$ for all $k_{1} \in \mathbb{Z}, \partial \in T$. 
Since $y_{j}+z_{1}+\alpha+x_{1}^{\prime} \notin M_{0}$ unless it is zero, then $y_{j}+z_{1}+\alpha+x_{1}^{\prime}, z_{0}$ are linearly independent unless $y_{j}+z_{1}+\alpha+x_{1}^{\prime}=0$. We can choose $\partial \in T$ such that $\partial\left(y_{j}+z_{1}+\right.$ $\left.x_{1}^{\prime}+\alpha\right)=0 \neq \partial\left(z_{0}\right)$. Applying this to (4.29) we get a contradiction. This contradiction shows that our assumption $b \neq b_{i}$ for some $i \in \mathbb{Z} \backslash\{0\}$ is not true. Thus, $b=b_{i}$ for all $i \in K$.

From Claim 3.1, for the fixed $j \in J$ we know that in (4.22) and (4.23),

$$
b=b_{i}, \quad \text { for all } i \in K,
$$

for any $x_{1}^{\prime} \in x_{1}+M_{0}, z_{0} \in M_{0}$ with $N_{0}=\mathbb{Z} x_{1}^{\prime}+\mathbb{Z} z_{0}$ being non-degenerate. Then, for any $x_{1}^{\prime} \in x_{1}+M_{0}, z_{0} \in M_{0}$ with $N_{0}=\mathbb{Z} x_{1}^{\prime}+\mathbb{Z} z_{0}$ being non-degenerate, we can choose $v_{x+y_{j}} \in V_{y_{j}+\alpha+x} \backslash\{0\}$ for any $x \in M_{1}=\mathbb{Z} x_{1}^{\prime}+M_{0}$ such that

$$
\left(t^{z} \partial\right) v_{x+y_{j}+z_{1}}=\partial\left(y_{j}+z_{1}+\alpha+x+b z\right) v_{x+z+z_{1}+y_{j}},
$$

for all $z_{1} \in M_{0}, x, z \in M_{0}$ or $x, z \in N_{0}$, with restriction $x+z+\alpha+y_{j} \neq 0 \neq x+y_{j}+$ $z_{1}+\alpha$ if $b \in\{0,1\}$, i.e.,

$$
\left(t^{z} \partial\right) v_{x+y_{j}}=\partial\left(y_{j}+\alpha+x+b z\right) v_{x+z+y_{j}}
$$

for all $z \in M_{0} \cup N_{0}, \quad x \in M_{0}+N_{0}=M_{1}$, with restriction $x+z+\alpha+y_{j} \neq 0 \neq$ $x+y_{j}+\alpha$ if $b \in\{0,1\}$.

Thus, we have chosen all $v_{x+y_{j}} \in V_{\alpha+x+y_{j}}$ for all $x \in M_{1}$, with $x+y_{j}+\alpha \neq 0$ if $b \in\{0,1\}$.

Claim 3.2. For a fixed $j \in J$,

$$
\left(t^{z} \partial\right) v_{x+y_{j}}=\partial\left(\alpha+y_{j}+x+b z\right) v_{y_{j}+x+z}
$$

for all $z, x \in M_{1}$, if $b \in\{0,1\}$ we need restrictions $x+y_{j}+\alpha \neq 0 \neq y_{j}+x+z+\alpha$.

If $z \in M_{0}$, (4.31) implies (4.32). Suppose $z \in M_{0}+N_{0} \backslash M_{0}$. Then we can choose $z_{1}^{\prime} \in$ $x_{1}+M_{0}, z_{2} \in M_{0}$ (since $M_{0}$ is non-degenerate) such that $\mathbb{Z} z_{1}^{\prime}+\mathbb{Z} z_{2}$ is non-degenerate and $z \in \mathbb{Z} z_{1}^{\prime}+\mathbb{Z} z_{2}$. From (4.31) we know that (4.32) holds for this $z$ also. Claim 3.2 follows.

From Claim 3.2, it follows that

$$
V\left(\alpha+y_{j}, M_{1}\right) \simeq \begin{cases}\mathscr{A}_{\alpha+y_{j}, b_{j}}\left(W\left(M_{1}, T, \varphi\right)\right), & \\ \mathscr{A}_{\beta}\left(W\left(M_{1}, T, \varphi\right)\right), & b_{0}=0, \\ \tilde{\mathscr{A}}_{\beta}\left(W\left(M_{1}, T, \varphi\right)\right), & b_{0}=0, \\ \mathscr{B}_{\beta}\left(W\left(M_{1}, T, \varphi\right)\right), & b_{0}=1, \\ \tilde{\mathscr{B}}_{\beta}\left(W\left(M_{1}, T, \varphi\right)\right), & b_{0}=1, \\ \mathscr{A}_{0, b_{0}}^{\prime}\left(W\left(M_{1}, T, \varphi\right)\right), & b_{0} \in\{0,1\}, \\ \mathscr{A}_{0, b_{0}}^{\prime}\left(W\left(M_{1}, T, \varphi\right)\right)+F v_{0}, & b_{0} \in\{0,1\} .\end{cases}
$$


The last six cases possibly occur only when $\alpha+y_{j} \in M_{1}$. Since $M_{1}=M_{0}+\mathbb{Z} x_{1} \neq M_{0}$, then (4.33) contradicts the maximality of $M_{0}$. Therefore we must have $A=M_{0}$, Claim 3 follows.

Next we suppose $\operatorname{dim} T \geq 3$.

Claim 4. For any two fixed linearly independent elements $\partial_{1}, \partial_{2} \in T$, there exist $b \in F$ and $v_{x} \in V_{\alpha+x}$ for all $x \in A$ such that

$$
\left(t^{x} \partial\right) v_{y}=\partial(y+\alpha+b x) v_{x+y}, \quad \text { for all } x, y \in A, \partial \in T_{0}
$$

with $y+\alpha \neq 0 \neq x+y+\alpha$ where $T_{0}=F \partial_{1}+F \partial_{2}$.

From Zorn's Lemma and Claim 3, there exists a maximal sub-group $B$ of $A$ so that there exist a sub-group $B_{1} \subset B, b \in F$ and $v_{x} \in V_{\alpha+x}$ for all $x \in B$ such that $\left.\varphi\right|_{T_{0} \times B_{1}}$ is non-degenerate, and that

$$
\left(t^{x} \partial\right) v_{y}=\partial(y+\alpha+b x) v_{x+y}, \quad \text { for all } x, y \in B, \partial \in T_{0}
$$

with $y+\alpha \neq 0 \neq x+y+\alpha$. We shall show that $B=A$. Otherwise we suppose that $A \neq B$.

Let $x_{3} \in A \backslash B$. For any $x_{1}, x_{2} \in B \backslash\{0\}$, we can choose $x_{1}^{\prime} \in B$ such that $\partial_{1}\left(x_{1}^{\prime}\right) \neq 0$ and $\operatorname{dim}_{F}\left(F x_{1}+F x_{1}^{\prime}\right)=\operatorname{dim}_{F}\left(F x_{2}+F x_{1}^{\prime}\right)=2$ in $T_{0}^{*}$. We choose $\partial_{3} \in T \backslash T_{0}$ (since $F$ is algebraically closed) such that

$$
\partial_{3}(x) \neq 0, \quad \forall x \in N_{1}=\sum_{i=1}^{3} \mathbb{Z} x_{i}+\mathbb{Z} x_{1}^{\prime} \backslash\{0\}
$$

and $\left.\varphi\right|_{\left(F \partial_{1}+F \partial_{3}\right) \times N_{0}}$ is non-degenerate where $N_{0}=\mathbb{Z} x_{1}+\mathbb{Z} x_{1}^{\prime}+\mathbb{Z} x_{2}$ is a free abelian group. Note that $\left.\varphi\right|_{\left(F \partial_{1}+F \partial_{3}\right) \times N_{1}}$ is non-degenerate. Since $F$ is algebraically closed, there exist distinct $\lambda_{1}, \lambda_{2}, \lambda_{3} \in F$ such that $\left.\operatorname{ker}\left(\partial_{2}+\lambda_{i} \partial_{3}\right)\right|_{N_{1}}=0$ and $\left.\varphi\right|_{T_{i} \times N_{0}}$ is non-degenerate, where $T_{i}=F \partial_{1}+F\left(\partial_{2}+\lambda_{i} \partial_{3}\right)$ for $i=1,2,3$. So $\left.\varphi\right|_{T_{i} \times N_{1}}$ is nondegenerate. Then $W_{i}=W\left(N_{1}, T_{i}, \varphi\right)$ are simple generalized Witt algebras. From Claim 3, corresponding to (4.35) we know that, for $i=1,2,3$,

$$
V\left(\alpha, N_{1}\right) \simeq \begin{cases}\mathscr{A}_{\alpha, b_{i}}\left(W_{i}\right), & \\ \mathscr{A}_{\beta_{i}}\left(W_{i}\right), & b_{i}=0, \\ \tilde{\mathscr{A}}_{\beta_{i}}\left(W_{i}\right), & b_{i}=0, \\ \mathscr{B}_{\beta_{i}}\left(W_{i}\right), & b_{i}=1, \\ \tilde{\mathscr{B}}_{\beta_{i}}\left(W_{i}\right), & b_{i}=1, \\ \mathscr{A}_{0, b_{i}}^{\prime}\left(W_{i}\right), & b_{i} \in\{0,1\}, \\ \mathscr{A}_{0, b_{i}}^{\prime}\left(W_{i}\right) \oplus F v_{0}, & b_{i} \in\{0,1\},\end{cases}
$$


where $b_{i} \in F, \beta_{i} \in T^{*}$. We know that $U=W\left(\mathbb{Z} x_{1}^{\prime}, F \partial_{1}, \varphi\right)$ is a centerless Virasoro algebra. From (4.37) we see that

$$
V\left(\alpha, Z x_{1}^{\prime}\right) \simeq \begin{cases}\mathscr{A}_{\alpha, b_{i}}(U), & \\ \mathscr{A}_{\beta_{i}}(U), & b_{i}=0, \\ \mathscr{B}_{\beta_{i}}(U), & b_{i}=1, \\ \mathscr{A}_{0, b_{i}}^{\prime}(U), & b_{i} \in\{0,1\}, \\ \mathscr{A}_{0, b_{i}^{\prime}}^{\prime}(U) \oplus F v_{0}, & b_{i} \in\{0,1\} .\end{cases}
$$

Using Theorem 3.2 we see that $b_{1}=b_{2}=b_{3}$ or $b_{1}, b_{2}, b_{3} \in\{0,1\}$. So two of $\left\{b_{1}, b_{2}, b_{3}\right\}$ are equal, say $b_{1}=b_{2}$, (actually we will see that $b_{1}=b_{2}=b_{3}$ ).

From (3.37) for $i=1$, we can choose $v_{x} \in V_{\alpha+x}$ for all $x \in N_{1}$ such that

$$
\left(t^{x} \partial\right) v_{y}=\partial\left(y+\alpha+b_{1} x\right) v_{x+y}, \quad \text { for all } x, y \in N_{1}, \partial \in T_{1},
$$

with $\alpha+x+y \neq 0 \neq \alpha+y$.

Since $N_{1}$ is of finite rank, we know that there exists a sub-group $N_{0}^{\prime} \subseteq N_{1}$ such that $\partial_{1}(x) \neq 0$ for any $N_{1}=N_{0}^{\prime} \oplus K_{0}$ where $K_{0}=\operatorname{ker}\left(\left.\partial_{1}\right|_{N_{1}}\right)$. Then $W\left(N_{0}^{\prime}, \partial_{1}, \varphi\right)$ is a simple generalized Witt algebra.

From (4.37) for $i=2$, we can choose $v_{x}^{\prime} \in V_{\alpha+x}$ for all $x \in N_{1}$ such that

(4.40) $v_{x}^{\prime}=v_{x}, \quad$ for all $x \in N_{0}^{\prime}, \quad$ and

$$
\left(t^{x} \partial\right) v_{y}^{\prime}=\partial\left(y+\alpha+b_{1} x\right) v_{x+y}^{\prime}, \quad \text { for all } x, y \in N_{1}, \partial \in T_{2},
$$

with $\alpha+x+y \neq 0 \neq \alpha+y$.

Claim 4.1. $v_{y}=v_{y}^{\prime}$ for all $y \in N_{1}$ with $\alpha+y \neq 0$.

From (4.39), (4.40) we know that

$$
\begin{aligned}
& \left(t^{x} \partial_{1}\right) v_{y}^{\prime}=\partial_{1}\left(y+\alpha+b_{1} x\right) v_{x+y}^{\prime} \quad \text { for all } x, y \in N_{1} \\
& \left(t^{x} \partial_{1}\right) v_{y}=\partial_{1}\left(y+\alpha+b_{1} x\right) v_{x+y}, \quad \text { for all } x, y \in N_{1}
\end{aligned}
$$

with $y+\alpha \neq 0 \neq y+x+\alpha$.

Case 1: $b_{1} \neq 0$

For any $x \in N_{1}$, there exists $y \in N_{0}^{\prime}$ such that $\partial_{1}\left(x+\alpha+b_{1}(y-x)\right) \neq 0$. From

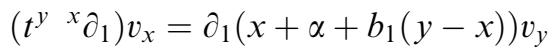

$$
\begin{aligned}
& =\partial_{1}\left(x+\alpha+b_{1}(y-x)\right) v_{y}^{\prime} \quad\left(\text { since } y \in N_{0}^{\prime}\right) \\
& =\left(\begin{array}{ll}
t^{y} & x \\
\partial_{1}
\end{array}\right) v_{x}^{\prime} \neq 0
\end{aligned}
$$

we obtain that $v_{x}^{\prime}=v_{x}$ for all $x \in N_{1}$ with $x+\alpha \neq 0$. Claim 4.1 follows in this case. 
Case 2: $b_{1}=0$

Choose $y_{0} \in N_{0}^{\prime}$ with $\partial_{1}\left(y_{0}+\alpha\right) \neq 0$. Then for any $x \in N_{1} \backslash\{0\}$ we deduce

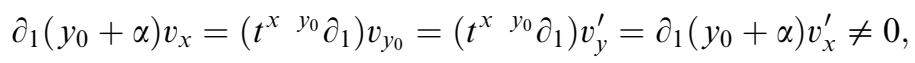

so $v_{x}=v_{x}^{\prime}$ for all $x \in N_{1}$ with $x+\alpha \neq 0$. Claim 4.1 also holds in this case. Therefore Claim 4.1 follows.

From Claim 4.1, Equations (4.39) and (4.40), we know that

$$
\left(t^{x} \partial\right) v_{y}=\partial\left(y+\alpha+b_{1} x\right) v_{x+y}, \quad \text { for all } x, y \in N_{1}, \partial \in \bigoplus_{i=1}^{3} F \partial_{i}
$$

with $\alpha+y \neq 0 \neq \alpha+x+y$. Therefore in (4.35) and (4.37)

$$
b=b_{1}=b_{2}=b_{3}
$$

Since $x_{1}, x_{2}$ run over $B$, since (4.43) is compatible for different $x_{1}, x_{2}$, we can choose $v_{x} \in V_{\alpha+x}$ for all $x \in B+\mathbb{Z} x_{3}$ such that

$$
\left(t^{x} \partial\right) v_{y}=\partial\left(y+\alpha+b_{1} x\right) v_{x+y}, \quad \text { for all } x, y \in B+\mathbb{Z} x_{3}, \partial \in T_{0},
$$

with $\alpha+y \neq 0 \neq \alpha+x+y$. A contradiction to the maximality of $B$. Claim 4 follows. From Claim 4, we know that there exists a maximal subspace $T^{\prime} \subset T$ with $\operatorname{dim} T^{\prime} \geq 2$ and with the property: we can choose $v_{x} \in V_{\alpha+x}$ for all $x \in A$ such that

$$
\left(t^{x} \partial\right) v_{y}=\partial(y+\alpha+b x) v_{x+y}, \quad \text { for all } x, y \in A, \partial \in T^{\prime}
$$

where $b \in F, y+\alpha \neq 0 \neq x+y+\alpha$.

It is sufficient to show that $T^{\prime}=T$. To the contrary, suppose $T^{\prime} \neq T$. Choose $x_{0}, y_{0} \in A$ such that $\left.\left(\alpha+y_{0}\right)\right|_{T^{\prime}}$ and $\left.x_{0}\right|_{T^{\prime}}$ are linearly independent over $F$. Then there exist $\partial_{1} \in T^{\prime}$ such that $\partial_{1}\left(y_{0}+\alpha+b x_{0}\right)=0 \neq \partial_{1}\left(x_{0}\right)$. Choose $\partial_{2} \in T \backslash T^{\prime}$.

From Claim 4, there exist $b^{\prime} \in F$, and $v_{x}^{\prime} \in V_{\alpha+x}$ for all $x \in A$ such that

$$
\left(t^{x} \partial\right) v_{y}^{\prime}=\partial\left(y+\alpha+b^{\prime} x\right) v_{x+y}^{\prime}, \quad \text { for all } x, y \in A, \partial \in F \partial_{1}+F \partial_{2}
$$

with $y+\alpha \neq 0 \neq \alpha+x+y$. From (4.47), (4.46) we see that

$$
\begin{aligned}
& \left(t^{x_{0}} \partial_{1}\right) v_{y_{0}}=\partial_{1}\left(y_{0}+\alpha+b x_{0}\right) v_{x_{0}+y_{0}}=0 \\
& \left(t^{x_{0}} \partial_{1}\right) v_{y_{0}}^{\prime}=\partial_{1}\left(y_{0}+\alpha+b^{\prime} x_{0}\right) v_{x_{0}+y_{0}}^{\prime}=\partial_{1}\left(x_{0}\right)\left(b^{\prime}-b\right) v_{x_{0}+y_{0}}^{\prime} .
\end{aligned}
$$

It is clear that $v_{y_{0}}^{\prime} \in F v_{y_{0}}$, so $\left(t^{x_{0}} \partial_{1}\right) v_{y_{0}}^{\prime} \in F\left(t^{x_{0}} \partial_{1}\right) v_{y_{0}}=0$. Applying this to (4.48) we deduce that $b^{\prime}=b$ in (4.46) and (4.47). 
Claim 5. In (4.46), (4.47), $v_{x}^{\prime}=a v_{x}$ for all $x \in A$ with $\alpha+x \neq 0$ where $a \in F^{*}$.

Suppose that $v_{x}^{\prime}=f(x) v_{x}$ for all $x \in A \backslash\{0\}$. From $t^{x} \partial_{1} v_{y}^{\prime}=\partial(y+\alpha+b x) v_{x+y}^{\prime}$, we deduce that

$$
\partial_{1}(y+\alpha+b x)(f(y)-f(x+y))=0, \quad \forall x, y \in A \backslash\{0\} .
$$

It is not difficult to obtain that

$$
f(x)=f(y), \quad \forall x, y \in A \backslash\{0\} .
$$

Therefore Claim 5 follows.

From (3.46), (3.47) and Claim 5, then, for $\tilde{T}=T^{\prime} \oplus F \partial_{2}$, there exists $v_{x} \in V_{\alpha+x}$ for all $x \in A$ such that

$$
\left(t^{x} \partial\right) v_{y}=\partial(y+\alpha+b x) v_{x+y}, \quad \text { for all } x, y \in A, \partial \in \tilde{T}
$$

with $y \neq \alpha \neq 0 \neq x+y+\alpha$. This contradicts the choice of $T^{\prime}$. Therefore $T^{\prime}=T$, i.e., (4.49) holds for all $\partial \in T$. From Lemma 3.5 we know that Theorem 4.1 follows.

\section{\$5 Classification over arbitrary field}

In Section 4 we classified weight representations with weight multiplicity 1 of simple generalized Witt algebras over algebraically closed field of characteristic 0 . Now we shall give the classification of indecomposable and irreducible weight representations with weight multiplicity 1 of simple generalized Witt algebras over an arbitrary field $F$ of characteristic 0 .

Theorem 5.1. Suppose $F$ is an arbitrary field of characteristic $0, W=W(A, T, \varphi)$ is a simple generalized Witt algebra. Let $V=\bigoplus_{x \in A} V_{\alpha+x}$ be a $W$-module, where $\beta \in T^{*}$,

$$
V_{\alpha+x}=\{v \in V \mid \partial v=\partial(\alpha+x) v, \text { for all } \partial \in T\}
$$

with $\operatorname{dim} V_{\alpha+x} \leq 1$. Then $V$ is isomorphic to one of the following for appropriate $\beta \in T^{*}$ and $b \in F$ :

(i) $\mathscr{A}_{\alpha, b}^{\prime}(W)$,

(ii) $\mathscr{A}_{0, b}^{\prime}(W) \oplus F v_{0}$ as direct sum of $W$-modules, where $b \in\{0,1\}$,

(iii) $\mathscr{A}_{\beta}(W), \tilde{\mathscr{A}}_{\beta}(W)$,

(iv) $\mathscr{B}_{\beta}(W), \tilde{\mathscr{B}}_{\beta}(W)$,

(v) $F v_{0}, 1$-dimensional trivial module,

(vi) $\mathscr{A}_{0, b}^{\prime}(W)$, where $b \in\{0,1\}$. 
Proof. Denote the algebraically closed extension of $F$ by $\bar{F}$. Consider the $\bar{F}$ extensions $\bar{V}=\bar{F} \otimes_{F} V, \bar{W}=\bar{F} \otimes_{F} W, \bar{V}_{\alpha+x}=\bar{F} \otimes_{F} V_{\alpha+x}$. Then $\bar{V}$ is a weight module with weight multiplicity 1 over $\bar{W}$. From Theorem 4.4 we see that there exist $b \in \bar{F}$ and $\beta \in \bar{T}^{*}$ such that $\bar{V}$ is isomorphic to one of the following: (i) $\mathscr{A}_{\alpha, b}(\bar{W})$, (ii) $\mathscr{A}_{\beta}(\bar{W}), \tilde{\mathscr{A}}_{\beta}(\bar{W})$, (iii) $\mathscr{B}_{\beta}(\bar{W}), \tilde{\mathscr{B}}_{\beta}(\bar{W})$, (iv) $\mathscr{A}_{0, b}^{\prime}(\bar{W}) \oplus F v_{0}$ as direct sum of $\bar{W}$ modules, where $b \in\{0,1\}$, (v) $\mathscr{A}_{0, b}(\bar{W})$, where $b \in\{0,1\}$, (vi) $F v_{0}$.

It suffices to show that $b \in F$ and $\beta \in T^{*}$.

Case (i): $\bar{V} \simeq \mathscr{A}_{\alpha, b}(\bar{W})$

Fix $\partial \in T \backslash\{0\}$. There exists $x \in A$ such that $\partial(x) \neq 0$. Then $W_{0}=W(\mathbb{Z} x, F \partial, \varphi)$ is a centerless Virasoro algebra over $F$, and $V(\mathbb{Z} x)=\bigoplus_{k \in \mathbb{Z}} V_{\alpha+k x}$ is a weight module with multiplicity 1 over $W_{0}$. From the hypothesis we see that

$$
\bar{V}(\mathbb{Z} x) \simeq \overline{\mathscr{A}}_{\alpha, b}\left(\bar{W}_{0}\right) .
$$

From Theorem 2.2 and (5.1) there exists $b^{\prime} \in F$ such that $V(\mathbb{Z} x) \simeq \mathscr{A}_{\alpha, b^{\prime}}\left(W_{0}\right)$. So

$$
\bar{V}(\mathbb{Z} x) \simeq \overline{\mathscr{A}}_{\alpha, b^{\prime}}\left(\bar{W}_{0}\right)
$$

Therefore $b=b^{\prime} \in F$ or $b, b^{\prime} \in\{0,1\} \subset F$.

Case (ii): $\bar{V} \simeq \mathscr{A}_{\beta}(\bar{W})$

In this case we may assume that $\alpha=0$. Fix an arbitrary $\partial \in T \backslash\{0\}$. There exists $x \in A$ such that $\partial(x) \neq 0$. Then $W_{0}=W(\mathbb{Z} x, F \partial, \varphi)$ is a centerless Virasoro algebra over $F$, and $V(\mathbb{Z} x)=\bigoplus_{k \in \mathbb{Z}} V_{\alpha+k x}$ is a weight module with multiplicity 1 over $W_{0}$. From the hypothesis we see that

$$
\bar{V}(\mathbb{Z} x) \simeq \overline{\mathscr{A}}_{\beta(\partial)}\left(\bar{W}_{0}\right)
$$

From Theorem 2.2 and (5.2) there exists $a \in F$ such that $V(\mathbb{Z} x) \simeq \mathscr{A}_{a}\left(W_{0}\right)$. So

$$
\bar{V}(\mathbb{Z} x) \simeq \overline{\mathscr{A}}_{a}\left(\bar{W}_{0}\right)
$$

Therefore $\beta(\partial)=a \in F$ for all $\partial \in T$, i.e., $\beta \in T^{*}$.

Case (iii): $\bar{V} \simeq \mathscr{A}_{\beta}(W)$

In this case the proof is the same as in Case (ii).

The other cases are quite clear.

As consequences, we have the following two theorems.

Theorem 5.2. Suppose $F$ is an arbitrary field of characteristic $0, W=W(A, T, \varphi)$ is a simple generalized Witt algebra. Let $V$ be a nontrivial irreducible weight module over $W$ with weight multiplicity 1 . Then $V \simeq \mathscr{A}_{\alpha, b}^{\prime}(W)$ for appropriate $\alpha \in T^{*}$ and $b \in F$. 
Theorem 5.3. Suppose $F$ is an arbitrary field of characteristic $0, W=W(A, T, \varphi)$ is a simple generalized Witt algebra. Let $V$ be a nontrivial indecomposable weight module over $W$ with weight multiplicity 1 . Then $V$ is isomorphic to only one of the following for appropriate $\beta \in T^{*}$ and $b \in F$ :

(i) $\mathscr{A}_{\beta, b}(W)$,

(ii) $\mathscr{A}_{\beta}(W), \tilde{\mathscr{A}}_{\beta}(W)$,

(iii) $\mathscr{B}_{\beta}(W), \tilde{\mathscr{B}}_{\beta}(W)$,

(iv) $\mathscr{A}_{0, b}^{\prime}(W)$, where $b \in\{0,1\}$.

\section{References}

[BB] Berman S. and Bilig Y.: Irreducible representations of toroidal Lie algebras in charac teristic zero. J. Algebra 221 (1999), 188231

[DZ1] Djokovic D. Z. and Zhao K.: Derivations, isomorphisms, and second cohomology of generalized Witt algebras. Trans. Amer. Math. Soc. 350 (1998), 643664

[DZ2] Djokovic D. Z. and Zhao K.: Generalized Cartan type $W$ Lie algebras in characteristic zero. J. Algebra 195 (1997), 170210

[E1] Eswara Rao S.: Irreducible representations of the Lie algebra of the diffeomorphisms of a $d$ dimensional torus. J. Algebra 182 (1996), 401421

[E2] Eswara Rao S.: Representations of Witt algebras. Publ. Res. Inst. Math. Sci. 30 (1994), 191201

[IK] Ikeda T. and Kawamoto N.: On the derivations of generalized Witt algebras over a field of characteristic zero. Hiroshima Math. J. 20 (1990), 4755

[J] Jia Y.: Weight representations of generalized Witt algebras; preprint, 1997

[Jo] Jordan D. A.: On the simplicity of Lie algebras of derivations of commutative algebras. J. Algebra 228 (2000), 580585

[Kp] Kaplansky I.: The Virasoro algebra. Comm. Math. Phys. 86 (1982), 4954

[M] Mathieu O.: Classification of Harish Chandra modules over the Virasoro algebra. Invent. Math. 107 (1992), 225234

[P] Passman D.: Simple Lie Algebras of Witt Type. J. Algebra 206 (1998), 682692

[PZ] Patera J. and Zassenhaus H.: The higher rank Virasoro algebras. Comm. Math. Phys. 136 (1991), 114

[SZ] Su Y. C. and Zhao K.: Generalized Virasoro and super Virasoro algebras and modules of intermediate series. J. Algebra 252 (2002), 119

[X] Xu X.: New generalized simple Lie algebras of Cartan type over a field with charac teristic 0. J. Algebra 224 (2000), 2358

Received August 20, 2002; revised and in final form February 10, 2003

Institute of Mathematics, Academy of Mathematics and System Sciences, Chinese Academy of

Sciences, Beijing 100080, P. R. China

kzhao@mail2.math.ac.cn 